\title{
Symmetries of projective spaces and spheres
}

Article

Accepted Version

Geher, G. (2020) Symmetries of projective spaces and spheres. International Mathematics Research Notices, 2020 (7). pp. 2205-2240. ISSN 1073-7928 doi: https://doi.org/10.1093/imrn/rny100 Available at https://centaur.reading.ac.uk/85681/

It is advisable to refer to the publisher's version if you intend to cite from the work. See Guidance on citing.

To link to this article DOI: http://dx.doi.org/10.1093/imrn/rny 100

Publisher: Oxford University Press

All outputs in CentAUR are protected by Intellectual Property Rights law, including copyright law. Copyright and IPR is retained by the creators or other copyright holders. Terms and conditions for use of this material are defined in the End User Agreement.

\section{www.reading.ac.uk/centaur}

\section{CentAUR}

Central Archive at the University of Reading

Reading's research outputs online 


\title{
SYMMETRIES OF PROJECTIVE SPACES AND SPHERES
}

\author{
GYÖRGY PÁL GEHÉR
}

\begin{abstract}
Let $H$ be either a complex inner product space of dimension at least two, or a real inner product space of dimension at least three, and let us fix an $\alpha \in\left(0, \frac{\pi}{2}\right)$. The purpose of this paper is to characterise all bijective transformations on the projective space $P(H)$ which preserve the quantum angle $\alpha$ (or Fubini-Study distance $\alpha$ ) between lines in both directions. (Let us emphasise that we do not assume anything about the preservation of other quantum angles). For real inner product spaces and when $H=\mathbb{C}^{2}$ we do this for every $\alpha$, and when $H$ is a complex inner product space of dimension at least three we describe the structure of such transformations for $\alpha \leq \frac{\pi}{4}$. Our result immediately gives an Uhlhorn-type generalisation of Wigner's theorem on quantum mechanical symmetry transformations, that is considered to be a cornerstone of the mathematical foundations of quantum mechanics. Namely, under the above assumptions, every bijective map on the set of pure states of a quantum mechanical system that preserves the transition probability $\cos ^{2} \alpha$ in both directions is a Wigner symmetry (thus automatically preserves all transition probabilities), except for the case when $H=\mathbb{C}^{2}$ and $\alpha=\frac{\pi}{4}$ where an additional possibility occurs. (Note that the classical theorem of Uhlhorn is the solution for the $\alpha=\frac{\pi}{2}$ case). Usually in the literature, results which are connected to Wigner's theorem are discussed under the assumption of completeness of $H$, however, here we shall remove this unnecessary hypothesis in our investigation. Our main tool is a characterisation of bijective maps on unit spheres of real inner product spaces which preserve one spherical angle in both directions.
\end{abstract}

\section{INTRODUCTION}

Characterising bijective isometries between normed spaces is a classical and important area of functional analysis. For instance, the Mazur-Ulam theorem asserts that every bijective isometry between real normed spaces is an affine map (i.e. a composition of a linear transformation and a translation by a vector). As a consequence, if two real normed spaces are isomorphic as metric spaces, they are also isomorphic as vector spaces. Another classical result in this area is the Banach-Stone theorem, which characterises bijective linear isometries between Banach spaces of continuous functions on compact Hausdorff spaces. In particular, the existence of such a map implies that the underlying compact Hausdorff spaces are topologically equivalent. Since the appearance of these remarkable theorems, the structures of bijective isometries have been studied and described for several

2010 Mathematics Subject Classification. Primary: 47B49, 51A05. Secondary: 47N50.

Key words and phrases. Projective space, quantum pure states, unit sphere, quantum angle preserving map, Fubini-Study metric, Wigner symmetry, transition probability preserving map, spherical angle preserving map. 
important normed spaces, and even today this is an active area of functional analysis. We refer to the books $[17,18]$ for an excellent introduction, and to the following papers: $[1,2,3,7,8,12,23,26,40]$.

Describing the structure of bijective isometries of non-linear spaces is also very important. One of the most classical examples of this is the famous Wigner's theorem on quantum mechanical symmetry transformations [5, 14, 19, 25, 28, 32, 39, 42]. First, let us state the theorem in its operator theoretic form, and then explain two equivalent rephrasing. Let $H$ be a complex (or real) Hilbert space, and $P_{1}(H)$ be the set of all rank-one orthogonal projections acting on $H$. In quantum mechanics rank-one orthogonal projections represent pure states of the system, and the transition probability between $P, Q \in P_{1}(H)$ is defined by $\operatorname{Tr} P Q \in[0,1]$, where $\operatorname{Tr}$ denotes the trace functional. Now, Wigner's theorem states the following:

Wigner's theorem. Let $H$ be a Hilbert space with $\operatorname{dim} H \geq 2$. Then every bijective map $\phi: P_{1}(H) \rightarrow P_{1}(H)$ which preserves the transition probability, i.e.

$$
\operatorname{Tr} \phi(P) \phi(Q)=\operatorname{Tr} P Q \quad\left(P, Q \in P_{1}(H)\right),
$$

is induced by a unitary or an antiunitary operator $U: H \rightarrow H$ (bijective orthogonal operator in the real case), i.e. we have:

$$
\phi(P)=U P U^{*} \quad\left(P \in P_{1}(H)\right) .
$$

Second, the following formula can be easily verified:

$$
\|P-Q\|=\sqrt{1-\operatorname{Tr} P Q} \quad\left(P, Q \in P_{1}(H)\right),
$$

where $\|\cdot\|$ denotes the usual operator norm. This quantity is called the gap distance of $P$ and $Q$. Therefore, Wigner's theorem characterises bijective isometries of $P_{1}(H)$ with respect to the gap metric. The gap metric was introduced and investigated by Sz.-Nagy and independently by Krein and Krasnoselski, and found applications in perturbation theory of linear operators, perturbation analysis of invariant subspaces, optimisation, robust control, etc. (see [20] for references).

Third, let $P(H)$ denote the projective space obtained from $H$, i.e. the space of all lines (one-dimensional subspaces) of $H$. Of course the map $P_{1}(H) \rightarrow P(H)$, $P \mapsto \operatorname{Im} P$ gives a natural bijection. If $0 \neq v \in H$ is a vector, then $[v]$ will stand for the line generated by it. The quantum angle or Fubini-Study distance between $[u],[v] \in P(H)$ is defined by the following formula:

$$
\measuredangle([u],[v]):=\arccos \frac{|\langle u, v\rangle|}{\|u\| \cdot\|v\|} \in\left[0, \frac{\pi}{2}\right] .
$$

We call a bijective isometry with respect to this distance a Wigner symmetry. In case when $\measuredangle([u],[v])=\frac{\pi}{2}$, then we will usually write $[u] \perp[v]$, moreover, given any subset $\mathcal{L} \subset P(H)$ we will use the notation $\mathcal{L}^{\perp}:=\{[w] \in P(H):[w] \perp[u], \forall[u] \in$ $\mathcal{L}\}$. By a straightforward calculation we infer the following equation:

$$
\operatorname{Tr} P Q=\left(\frac{|\langle u, v\rangle|}{\|u\| \cdot\|v\|}\right)^{2}=\cos ^{2} \measuredangle([u],[v])
$$

for every $P, Q \in P_{1}(H)$ such that $[u]=\operatorname{Im} P$ and $[v]=\operatorname{Im} Q$. Therefore, Wigner's theorem can be viewed as a characterisation of Wigner symmetries.

There is a huge literature of generalising Wigner's theorem in several directions, perhaps the first of them was Uhlhorn's theorem [41] which we state now. 
Uhlhorn's theorem. Let $H$ be a Hilbert space with $\operatorname{dim} H \geq 3$ and $\phi: P(H) \rightarrow$ $P(H)$ be a bijective map preserving orthogonality in both directions, i.e.

$$
\phi([u]) \perp \phi([v]) \Longleftrightarrow[u] \perp[v] \quad([u],[v] \in P(H)) .
$$

Then $\phi$ is a Wigner symmetry, hence there exists a unitary or an antiunitary operator $U: H \rightarrow H$ (bijective orthogonal operator in the real case) such that

$$
\phi([u])=[U u] \quad([u] \in P(H)) .
$$

This was a serious improvement, since in Uhlhorn's theorem only the preservation of the quantum logical structure is assumed, while in Wigner's theorem its complete probabilistic structure is preserved. We point out that Uhlhorn's theorem can be stated also for non-complete inner product spaces (see Lemma 3.8), which can be found e.g. in [16]. For further generalisations of Wigner's theorem on projective spaces we mention $[10,11,13,14,27,30,33,34,35,36]$, and on Grassmann spaces or certain classes of idempotent operators we refer to [9, 20,21, 22, 29, 37, 38].

The present paper is devoted to provide a very natural generalisation of Wigner's theorem, following Uhlhorn's direction. Instead of preserving orthogonality of lines in both directions, we will assume that a fixed quantum angle $\alpha \in\left(0, \frac{\pi}{2}\right)$ is preserved in both directions. We point out that this problem has been solved partially in [24] by Li, Plevnik and Šemrl, namely, they proved that if $\alpha \leq \frac{\pi}{4}$ and $H$ is a real space with $5 \leq \operatorname{dim} H<\infty$, then such maps are always Wigner symmetries. Their method depends heavily on these rather restrictive assumptions. Here, by developing a novel technique, we solve this problem in a much more general setting. Namely, in the real case we will answer the question completely, i.e. for every (not necessarily complete) inner product space with dimension at least three and for all quantum angles $\alpha$. We will also solve the problem for every quantum angle $\alpha$ in the pure qubit case, i.e. when $H=\mathbb{C}^{2}$, which could be quite a surprise since Uhlhorn's theorem obviously does not hold in two dimensions. For complex inner product spaces of dimension at least three we will provide a characterisation for quantum angles $\alpha \leq \frac{\pi}{4}$.

The outline of the paper is the following: in the next section we state four theorems which are our main results. Then in Section 3 we will prove a result about transformations on spheres preserving one spherical angle between unit vectors in both directions. This will put us in the position to prove our Uhlhorn-type generalisation of Wigner's theorem in the real case which will be given in Section 4 , and in the same section we will also prove the complex case.

\section{Statements of the main Results}

Let $H$ be an inner product space. If the vectors $x, y \in H$ are orthogonal, then we will write $x \perp y$. By Rätz's version of Wigner's theorem [32], if $H$ is a real inner product space, then a Wigner symmetry $\phi: P(H) \rightarrow P(H)$ is always induced by a bijective linear isometry $O: H \rightarrow H$, i.e. we have

$$
\phi([v])=[O v] \quad([v] \in P(H)) .
$$

Moreover, in the complex case every Wigner symmetry $\phi: P(H) \rightarrow P(H)$ is induced by a bijective linear or conjugatelinear isometry $U: H \rightarrow H$ :

$$
\phi([v])=[U v] \quad([v] \in P(H)) .
$$

Now, we state our generalisation of Wigner's theorem for real spaces. 
Theorem 1. Let $H$ be a real inner product space with $\operatorname{dim} H \geq 3$ and suppose that $0<\alpha<\frac{\pi}{2}$. If $\phi: P(H) \rightarrow P(H)$ is a bijective map which preserves the quantum angle $\alpha$ between lines in both directions, i.e. $\phi$ satisfies

$$
\measuredangle([u],[v])=\alpha \Longleftrightarrow \measuredangle(\phi([u]), \phi([v]))=\alpha \quad([u],[v] \in P(H)),
$$

then $\phi$ is a Wigner symmetry, hence possesses the form (1).

The proof of Theorem 1 will require the study of bijective transformations on the unit sphere $S_{H}=\{h \in\|h\|=1\}$ of a real inner product space $H$ which preserve one spherical angle. The spherical angle between two unit vectors $x, y \in S_{H}$ is

$$
\varangle(x, y):=\arccos \langle x, y\rangle \in[0, \pi],
$$

which also provides the geodesic distance on the sphere $S_{H}$. (Note that writing $\varangle(x, y)=\frac{\pi}{2}$ is the same as $\left.x \perp y\right)$. The study of such maps was initiated by Everling in [15], where he proved a result, similar to Theorem 2, under the assumption that $3 \leq \operatorname{dim} H<\infty$ and $\alpha \leq \frac{\pi}{2}$. Apparently, he was motivated by the surprising Beckman-Quarles theorem [6] which asserts that every map on $H$ preserving distance 1 is automatically an isometry if we have $2 \leq \operatorname{dim} H<\infty$.

We state our improvement of Everling's theorem below.

Theorem 2. Let $H$ be a real inner product space with $3 \leq \operatorname{dim} H$, and $0<\alpha<$ $\pi$. Assume that $\psi: S_{H} \rightarrow S_{H}$ is a bijective transformation which preserves the spherical angle $\alpha$ in both directions, i.e. we have

$$
\varangle(x, y)=\alpha \Longleftrightarrow \varangle(\psi(x), \psi(y))=\alpha \quad\left(x, y \in S_{H}\right) .
$$

Then there exists a bijective linear isometry $R: H \rightarrow H$ such that we have

(i) either

$$
\psi(x)=R x \quad\left(x \in S_{H}\right),
$$

(ii) or $\alpha=\frac{\pi}{2}$ and

$$
\psi(x) \in\{-R x, R x\} \quad\left(x \in S_{H}\right) .
$$

Next, let $H=\mathbb{C}^{2}$. For any $[u] \in P\left(\mathbb{C}^{2}\right)$ the symbol $[u]^{\perp}$ stands for the unique line orthogonal to $[u]$. Now, we state our theorem about pure qubit states.

Theorem 3. Suppose that $0<\alpha<\frac{\pi}{2}$. If $\phi: P\left(\mathbb{C}^{2}\right) \rightarrow P\left(\mathbb{C}^{2}\right)$ is a bijective map preserving the quantum angle $\alpha$ in both directions, i.e.

$$
\measuredangle([u],[v])=\alpha \Longleftrightarrow \measuredangle(\phi([u]), \phi([v]))=\alpha \quad\left([u],[v] \in P\left(\mathbb{C}^{2}\right)\right),
$$

then

(i) either $\phi$ is a Wigner symmetry, hence possessing the form (2),

(ii) or $\alpha=\frac{\pi}{4}$ and there exists a Wigner symmetry $\widetilde{\phi}$ such that

$$
\phi([v]) \in\left\{\widetilde{\phi}([v]), \widetilde{\phi}([v])^{\perp}\right\} \quad\left([v] \in P\left(\mathbb{C}^{2}\right)\right) .
$$

Finally, our last result is a generalisation of Wigner's theorem for complex inner product spaces, which we state below.

Theorem 4. Suppose that $H$ is a complex inner product space, $3 \leq \operatorname{dim} H$ and $0<\alpha \leq \frac{\pi}{4}$. Assume that $\phi: P(H) \rightarrow P(H)$ is a bijective map which satisfies

$$
\measuredangle([u],[v])=\alpha \Longleftrightarrow \measuredangle(\phi([u]), \phi([v]))=\alpha \quad([u],[v] \in P(H)) .
$$

Then $\phi$ is a Wigner symmetry, therefore possesses the form (2). 


\section{SyMmetries OF SPHERES}

The aim of this section is to verify Theorem 2, but before that we need to prove several lemmas. Note that although our method follows some of Everling's ideas, it essentially differs from his approach at several points. Let $H$ be a real inner product space of dimension at least three. A map $\psi: S_{H} \rightarrow S_{H}$ is said to be a $\varangle$-isometry if we have

$$
\varangle(x, y)=\varangle(\psi(x), \psi(y)) \quad\left(x, y \in S_{H}\right),
$$

or equivalently

$$
\langle x, y\rangle=\langle\psi(x), \psi(y)\rangle \quad\left(x, y \in S_{H}\right) .
$$

We begin with the following characterisation of bijective $\varangle$-isometries, which is probably well-known.

Lemma 3.1. Let $H$ be a real inner product space with $\operatorname{dim} H \geq 3$. If $\psi: S_{H} \rightarrow S_{H}$ is a bijective $\varangle$-isometry, then (3) is satisfied with a bijective linear isometry $R$.

Proof. We define the following bijective map:

$$
R: H \rightarrow H, \quad R x=\left\{\begin{array}{cl}
\|x\| \cdot \psi\left(\frac{1}{\|x\|} x\right) & \text { if } x \neq 0 \\
0 & \text { if } x=0
\end{array}\right.
$$

We have to show that $R$ is a linear isometry. We obviously have $\langle R x, R y\rangle=\langle x, y\rangle$ $(x, y \in H)$. Therefore we obtain $\|R x-R y\|^{2}=\langle R x, R x\rangle+\langle R y, R y\rangle-2\langle R x, R y\rangle=$ $\langle x, x\rangle+\langle y, y\rangle-2\langle x, y\rangle=\|x-y\|^{2}$, hence $R$ is an isometry. By the famous MazurUlam theorem we immediately obtain the linearity of $R$.

For some vectors $z_{1}, \ldots z_{n} \in H$ the symbol $\left[z_{1}, \ldots z_{n}\right]$ will stand for the generated subspace in $H$. We will use the following notation for an $x \in S_{H}$ :

$$
x^{(\alpha)}:=\left\{y \in S_{H}: \varangle(x, y)=\alpha\right\} .
$$

Next, we investigate the set $x^{(\alpha)} \cap y^{(\beta)}$ which later will be utilised in order to obtain further spherical angles which $\psi$ preserves.

Lemma 3.2. Let $H$ be a real inner product space with $\operatorname{dim} H \geq 3$. Let $x, y \in S_{H}$, $0<\alpha<\beta<\pi$, and assume that $\gamma:=\varangle(x, y) \in(0, \pi]$. Then the following equivalences are satisfied:

$$
x^{(\alpha)} \cap y^{(\beta)}=\emptyset \Longleftrightarrow\left\{\begin{array}{cl}
\gamma>\alpha+\beta \text { or } \gamma<\beta-\alpha, & \text { if } \alpha+\beta<\pi, \\
\gamma<\beta-\alpha, & \text { if } \alpha+\beta=\pi, \\
\gamma>2 \pi-\alpha-\beta \text { or } \gamma<\beta-\alpha, & \text { if } \alpha+\beta>\pi,
\end{array}\right.
$$

and

$$
\#\left(x^{(\alpha)} \cap y^{(\beta)}\right)=1 \Longleftrightarrow\left\{\begin{array}{cl}
\gamma \in\{\alpha+\beta, \beta-\alpha\}, & \text { if } \alpha+\beta<\pi, \\
\gamma=\beta-\alpha, & \text { if } \alpha+\beta=\pi, \\
\gamma \in\{2 \pi-\alpha-\beta, \beta-\alpha\}, & \text { if } \alpha+\beta>\pi .
\end{array}\right.
$$

Moreover, if $\alpha \neq \frac{\pi}{2}$, then we have

and

$$
x^{(\alpha)} \cap y^{(\alpha)}=\emptyset \Longleftrightarrow\left\{\begin{array}{cc}
\gamma>2 \alpha, & \text { if } \alpha<\frac{\pi}{2}, \\
\gamma>2 \pi-2 \alpha, & \text { if } \alpha>\frac{\pi}{2},
\end{array}\right.
$$

$$
\#\left(x^{(\alpha)} \cap y^{(\alpha)}\right)=1 \Longleftrightarrow\left\{\begin{array}{cl}
\gamma=2 \alpha, & \text { if } \alpha<\frac{\pi}{2} \\
\gamma=2 \pi-2 \alpha, & \text { if } \alpha>\frac{\pi}{2} .
\end{array}\right.
$$


Proof. We will only consider the first two equivalences, since the other two can be verified similarly. Let us observe that the quantity $\#\left(x^{(\alpha)} \cap y^{(\beta)} \cap K\right)$ is the same for every three-dimensional subspace $K$ of $H$ which contains $x$ and $y$. Thus we have $x^{(\alpha)} \cap y^{(\beta)}=\emptyset$ if and only if we have $x^{(\alpha)} \cap y^{(\beta)} \cap K=\emptyset$ for some (or equivalently, for all) such $K$. Moreover, if $\#\left(x^{(\alpha)} \cap y^{(\beta)} \cap K\right)=1$ with such a $K$, and this unique element is denoted by $u$, then the symmetry of the sphere implies $u \in[x, y]$. Therefore we conclude that $\#\left(x^{(\alpha)} \cap y^{(\beta)}\right)=1$ if and only if we have $\#\left(x^{(\alpha)} \cap y^{(\beta)} \cap K\right)=1$ for some (or equivalently, for all) three-dimensional subspaces $K$ containing $x$ and $y$. In particular this cannot happen if $\gamma=\pi$, since in this case we have $[x, y]=[x]$ and thus $u \in\{x, y\}$, which is a contradiction. Therefore we only have to prove our statement for the case when $\operatorname{dim} H=3$ and $\gamma<\pi$, so from now on we will assume this.

The first spherical law of cosines implies that $x^{(\alpha)} \cap y^{(\beta)} \neq \emptyset$ if and only if there exists a $C \in[0, \pi]$ such that

$$
\cos \gamma=\cos \alpha \cos \beta+\sin \alpha \sin \beta \cos C .
$$

This is equivalent to $\cos \gamma \in[\cos (\alpha+\beta), \cos (\beta-\alpha)]=[\cos (2 \pi-\alpha-\beta), \cos (\beta-\alpha)]$, which yields (4).

Clearly, $\#\left(x^{(\alpha)} \cap y^{(\beta)}\right)=1$ implies $C \in\{0, \pi\}$, therefore we conclude that $\#\left(x^{(\alpha)} \cap y^{(\beta)}\right)=1$ holds exactly when one of the following two conditions is satisfied:

- $C=0$ and $\gamma=\beta-\alpha$, or

- $C=\pi, \alpha+\beta \neq \pi$ and $\gamma=\left\{\begin{array}{cc}\alpha+\beta, & \text { if } \alpha+\beta<\pi, \\ 2 \pi-\alpha-\beta, & \text { if } \alpha+\beta>\pi .\end{array}\right.$

The equivalences stated in the lemma follows from this.

It is a standard and natural method in the theory of preservers that one characterises a relation in terms of the property which is preserved by our bijective map in both directions, and then concludes that this map also preserves this relation in both directions. Here this method will be utilised several times.

We proceed with the verifications of the following two lemmas.

Lemma 3.3. Let $0<\alpha<\frac{\pi}{2}$. Under the hypotheses of Theorem 2 we have the following two properties:

$$
\varangle(x, y)=j \alpha \Longleftrightarrow \varangle(\psi(x), \psi(y))=j \alpha \quad\left(x, y \in S_{H}, j \in \mathbb{N}, 2 \leq j<\frac{\pi}{\alpha}\right) .
$$

and

$$
\varangle(x, y) \leq j \alpha \Longleftrightarrow \varangle(\psi(x), \psi(y)) \leq j \alpha \quad\left(x, y \in S_{H}, j \in \mathbb{N}, 2 \leq j<\frac{\pi}{\alpha}\right)
$$

Proof. Assume that $j \in \mathbb{N}$ and $2 \leq j<\frac{\pi}{\alpha}$. It is straightforward that we have $\varangle(x, y)=j \alpha$ if and only if there is a unique $(j-1)$-element sequence $\left\{x_{1}, \ldots x_{j-1}\right\}$ such that $\varangle\left(x_{l}, x_{l+1}\right)=\varangle\left(x, x_{1}\right)=\varangle\left(x_{j-1}, y\right)=\alpha(l=1, \ldots j-2)$. Since $\psi$ and $\psi^{-1}$ are bijective transformations preserving the spherical angle $\alpha$ in both directions, we conclude that the latter condition is equivalent to the existence of a unique $(j-1)$-element sequence $\left\{y_{1}, \ldots y_{j-1}\right\}$ such that $\varangle\left(y_{l}, y_{l+1}\right)=\varangle\left(\psi(x), y_{1}\right)=$ $\varangle\left(y_{j-1}, \psi(y)\right)=\alpha(l=1, \ldots j-2)$. But this holds exactly when $\varangle(\psi(x), \psi(y))=j \alpha$, therefore we obtain (6).

The proof of (7) is very similar, we only have to observe that $\varangle(x, y) \leq j \alpha$ is satisfied if and only if there exists a $(j-1)$-element sequence $\left\{x_{1}, \ldots x_{j-1}\right\}$ such that $\varangle\left(x_{l}, x_{l+1}\right)=\varangle\left(x, x_{1}\right)=\varangle\left(x_{j-1}, y\right)=\alpha(l=1, \ldots j-2)$. 
Lemma 3.4. Let $H$ be a real inner product space with $\operatorname{dim} H \geq 3$, and $\psi: S_{H} \rightarrow S_{H}$ be a bijective map. Assume that $0<\alpha<\frac{\pi}{2}$ and that $\psi$ satisfies

$$
\varangle(x, y) \leq \alpha \Longleftrightarrow \varangle(\psi(x), \psi(y)) \leq \alpha \quad\left(x, y \in S_{H}\right) .
$$

Then $\psi$ also fulfils the following:

$$
\varangle(x, y)=2 \alpha \Longleftrightarrow \varangle(\psi(x), \psi(y))=2 \alpha \quad\left(x, y \in S_{H}\right) .
$$

Proof. We only have to observe that $\varangle(x, y)=2 \alpha$ holds if and only if there is a unique $u \in S_{H}$ with $\varangle(x, u) \leq \alpha$ and $\varangle(u, y) \leq \alpha$.

In the previous lemma we could have shown the property (6) as well however, we will not need it in the sequel. Next, we show that the $\varangle$-isometriness of $\psi$ is a consequence of a much milder assumption.

Lemma 3.5. Let $H$ be a real inner product space with $\operatorname{dim} H \geq 3$, and $\psi: S_{H} \rightarrow S_{H}$ be a bijective map. Suppose that there exists a decreasing sequence $\left\{\alpha_{n}\right\}_{n=1}^{\infty} \subseteq(0, \pi)$ of spherical angles with $\lim _{n \rightarrow \infty} \alpha_{n}=0$ such that $\psi$ preserves all of them in both directions. Then $\psi$ is a $\varangle$-isometry.

Proof. From (7) of Lemma 3.3 we conclude that

$$
(j-1) \alpha_{n}<\varangle(x, y) \leq j \alpha_{n} \Longleftrightarrow(j-1) \alpha_{n}<\varangle(\psi(x), \psi(y)) \leq j \alpha_{n}
$$

holds for every $x, y \in S_{H}$ and $j, n \in \mathbb{N}, 3 \leq j<\frac{\pi}{\alpha_{n}}$. Since $\alpha_{n}$ can be arbitrarily small, we obtain that $\psi$ preserves every spherical angle which is less than $\pi$ in both directions. Hence we also have

$$
\varangle(x, y)<\pi \Longleftrightarrow \varangle(\psi(x), \psi(y))<\pi,
$$

and thus a negation of this equivalence gives that the spherical angle $\pi$ is also preserved in both directions, which completes the proof.

Using Lemma 3.2 we can provide other spherical angles which $\psi$ preserves.

Lemma 3.6. Let $H$ be a real inner product space with $\operatorname{dim} H \geq 3,0<\alpha<\beta<\pi$, $\alpha+\beta \leq \pi$, and $\psi: S_{H} \rightarrow S_{H}$ be a bijective map. Suppose that $\psi$ preserves the spherical angles $\alpha$ and $\beta$ in both directions. Then

(i) $\psi$ preserves the spherical angles $\beta-\alpha$ and $\alpha+\beta$ in both directions, if $\alpha+\beta<\pi$,

(ii) $\psi$ preserves the spherical angle $\beta-\alpha$ in both directions, if $\alpha+\beta=\pi$.

Proof. (i): Let us observe that the properties of $\psi$ implies $\psi\left(x^{(\alpha)} \cap y^{(\beta)}\right)=$ $\psi(x)^{(\alpha)} \cap \psi(y)^{(\beta)}$. Therefore, by Lemma 3.2 we have

$$
\begin{aligned}
\varangle(x, y) & \in\{\beta-\alpha, \alpha+\beta\} \Longleftrightarrow \#\left(x^{(\alpha)} \cap y^{(\beta)}\right)=1 \\
& \Longleftrightarrow \#\left(\psi(x)^{(\alpha)} \cap \psi(y)^{(\beta)}\right)=1 \Longleftrightarrow \varangle(\psi(x), \psi(y)) \in\{\beta-\alpha, \alpha+\beta\} .
\end{aligned}
$$

We observe that $\alpha<\frac{\pi}{2}$. Clearly, there exists a $j \in \mathbb{N}, 2 \leq j<\frac{\pi}{\alpha}$ such that $\beta-\alpha<j \alpha<\alpha+\beta$. Since

$$
\varangle(x, y)=\beta-\alpha \Longleftrightarrow \varangle(x, y) \in\{\beta-\alpha, \alpha+\beta\} \text { and } \varangle(x, y) \leq j \alpha,
$$

the above equivalence and (7) of Lemma 3.3 imply that $\psi$ preserves the spherical angle $\beta-\alpha$, and thus also $\alpha+\beta$ in both directions.

(ii): This is immediate from Lemma 3.2 . 
For a given set $\mathcal{A} \subseteq S_{H}$ we will use the notation $\operatorname{diam}_{\varangle}(\mathcal{A})=\sup \{\varangle(x, y): x, y \in$ $\mathcal{A}\}$. We proceed with the following crucial statement.

Lemma 3.7. Let us assume that the hypotheses of Theorem 2 are satisfied for $\psi$ and that we have $0<\alpha<\frac{\pi}{2}$. Then $\arccos \left(\frac{4 \cos ^{2} \alpha}{\cos \alpha+1}-1\right) \in(\alpha, 2 \alpha)$ holds, and $\psi$ preserves this spherical angle in both directions.

Proof. Let $x, y \in S_{H}$, and set $\gamma:=\varangle(x, y)$ and $h(\gamma):=\operatorname{diam}_{\varangle}\left(x^{(\alpha)} \cap y^{(\alpha)}\right)$. First, let us prove that $h(\gamma)=\alpha$ holds if and only if $\gamma=\arccos \left(\frac{4 \cos ^{2} \alpha}{\cos \alpha+1}-1\right)$. Clearly, we have $h(0)=2 \alpha$. By this and Lemma 3.2, the equation $h(\gamma)=\alpha$ implies $0<\gamma<$ $2 \alpha(<\pi)$. Let us consider a vector $u \in x^{(\alpha)} \cap y^{(\alpha)}$. Then there exists an orthonormal system $\left\{e_{1}, e_{2}, e_{3}\right\} \subset H$ and $\delta, \varepsilon \in[0, \pi]$ such that we have $x=\cos \frac{\gamma}{2} \cdot e_{1}+\sin \frac{\gamma}{2} \cdot e_{2}$, $y=\cos \frac{\gamma}{2} \cdot e_{1}-\sin \frac{\gamma}{2} \cdot e_{2}$ and $u=\cos \delta \cdot e_{1}+\sin \delta \cos \varepsilon \cdot e_{2}+\sin \delta \sin \varepsilon \cdot e_{3}$, furthermore, the following two equations are valid:

$$
\langle u, x\rangle=\cos \frac{\gamma}{2} \cos \delta+\sin \frac{\gamma}{2} \sin \delta \cos \varepsilon=\cos \alpha
$$

and

$$
\langle u, y\rangle=\cos \frac{\gamma}{2} \cos \delta-\sin \frac{\gamma}{2} \sin \delta \cos \varepsilon=\cos \alpha .
$$

Thus we conclude that $\delta \in\{0, \pi\}$ or $\varepsilon=\frac{\pi}{2}$. It is easy to see that the first possibility cannot happen. Indeed, in that case we would get $u \in\left\{e_{1},-e_{1}\right\}$, whence $\alpha=$ $\varangle(u, x)=\varangle(u, y) \in\left\{\frac{\gamma}{2}, \pi-\frac{\gamma}{2}\right\}$ would follow, that is a contradiction. In the second case we have $\cos \delta=\frac{\cos \alpha}{\cos (\gamma / 2)} \in(0,1)$, therefore we obtain the following equation:

$$
x^{(\alpha)} \cap y^{(\alpha)}=\left\{\frac{\cos \alpha}{\cos \frac{\gamma}{2}} \cdot e_{1}+\sqrt{1-\frac{\cos ^{2} \alpha}{\cos ^{2} \frac{\gamma}{2}}} \cdot \widetilde{e_{3}}: \widetilde{e_{3}} \in S_{H}, \widetilde{e_{3}} \perp e_{1}, \widetilde{e_{3}} \perp e_{2}\right\} .
$$

It is straightforward that

$$
h(\gamma)=\arccos \left(2 \frac{\cos ^{2} \alpha}{\cos ^{2} \frac{\gamma}{2}}-1\right) .
$$

One easily computes the only solution $\gamma_{0} \in(0,2 \alpha)$ of the equation $h\left(\gamma_{0}\right)=\alpha$ :

$$
\gamma_{0}=\arccos \left(\frac{4 \cos ^{2} \alpha}{\cos \alpha+1}-1\right) \text {. }
$$

Next, we show that $\gamma_{0}>\alpha$. Observe that the function $h:(0,2 \alpha) \rightarrow(0,2 \alpha)$ is a strictly decreasing bijection. Since we have

$$
\begin{aligned}
h(\alpha)>\alpha & \Longleftrightarrow \frac{2 \cos ^{2} \alpha}{\cos ^{2} \frac{\alpha}{2}}-1<\cos \alpha \Longleftrightarrow \cos \alpha \frac{4 \cos ^{2} \frac{\alpha}{2}-2}{\cos ^{2} \frac{\alpha}{2}}-1<\cos \alpha \\
& \Longleftrightarrow \cos \alpha \frac{3 \cos ^{2} \frac{\alpha}{2}-2}{\cos ^{2} \frac{\alpha}{2}}<1 \Longleftrightarrow\left(3-\frac{2}{\cos ^{2} \frac{\alpha}{2}}\right) \cos \alpha<1,
\end{aligned}
$$

and this latter inequality is satisfied, we obtain that $h(\alpha)>\alpha$ holds. Therefore, the monotonicity of $h$ implies $\gamma_{0}>\alpha$.

Now, by (10), if $u \in x^{(\alpha)} \cap y^{(\alpha)}$ and $\vartheta>0$, then we have $\#\left(x^{(\alpha)} \cap y^{(\alpha)} \cap u^{(\vartheta)}\right)=1$ if and only if $\vartheta=h(\gamma)$. In particular, we have $\#\left(x^{(\alpha)} \cap y^{(\alpha)} \cap u^{(\alpha)}\right)=1$ for every $u \in x^{(\alpha)} \cap y^{(\alpha)}$ exactly when $\gamma=\gamma_{0}$, which gives a characterisation of the spherical 
angle $\gamma_{0}$ in terms of $\alpha$. Therefore, similarly as in Lemma 3.6, we obtain that $\psi$ preserves the spherical angle $\gamma_{0}$ in both directions. Namely, we have

$$
\begin{aligned}
\varangle(x, y) & =\gamma_{0} \Longleftrightarrow \#\left(x^{(\alpha)} \cap y^{(\alpha)} \cap u^{(\alpha)}\right)=1 \text { for every } u \in x^{(\alpha)} \cap y^{(\alpha)} \\
& \Longleftrightarrow \#\left(\psi(x)^{(\alpha)} \cap \psi(y)^{(\alpha)} \cap \psi(u)^{(\alpha)}\right)=1 \text { for every } \psi(u) \in \psi(x)^{(\alpha)} \cap \psi(y)^{(\alpha)} \\
& \Longleftrightarrow \#\left(\psi(x)^{(\alpha)} \cap \psi(y)^{(\alpha)} \cap v^{(\alpha)}\right)=1 \text { for every } v \in \psi(x)^{(\alpha)} \cap \psi(y)^{(\alpha)} \\
& \varangle(\psi(x), \psi(y))=\gamma_{0},
\end{aligned}
$$

and this completes our proof.

Before we prove Theorem 2, we need Uhlhorn's theorem for (not necessarily complete) inner product spaces which we state below and that was proven e.g. in [16, Corollary 4.5] (however, in order to get the full statement, one may combine Theorem 4.1 and Corollary 4.5 of [16]).

Lemma 3.8 (Uhlhorn's theorem for inner product spaces). Let $H$ be a real or complex inner product space with $\operatorname{dim} H \geq 3$. Let $\phi: P(H) \rightarrow P(H)$ be a bijective map which preserves orthogonality in both directions, i.e.

$$
[u] \perp[v] \Longleftrightarrow \phi([u]) \perp \phi([v]) \quad([u],[v] \in P(H)) .
$$

Then $\phi$ is a Wigner symmetry, hence possesses the form (1) in the real case, and (2) in the complex case.

Now, we are in the position to verify our main result about symmetry transformations on unit spheres of real inner product spaces.

Proof of Theorem 2. The $\alpha=\frac{\pi}{2}$ case is a direct consequence Lemma 3.8. We will consider several possibilities separately in order to handle the remaining case.

Case 1: when $\alpha \leq \frac{\pi}{4}$. By Lemmas 3.3 and 3.7, our map $\psi$ preserves the spherical angles $2 \alpha$ and $\beta:=\arccos \left(\frac{4 \cos ^{2} \alpha}{\cos \alpha+1}-1\right) \in(\alpha, 2 \alpha)$ in both directions. Since $\alpha+\beta<2 \alpha+\beta<4 \alpha \leq \pi$, an application of Lemma 3.6 gives that $\psi$ preserves both of the positive spherical angles $2 \alpha-\beta$ and $\beta-\alpha$ in both directions. Clearly, one of these spherical angles is less than or equal to $\frac{\alpha}{2}$. Therefore an iteration gives us a decreasing sequence of positive spherical angles converging to zero such that $\psi$ preserves all of them in both directions. Applying Lemma 3.5 completes the proof of this case.

Case 2: when $\frac{\pi}{4}<\alpha<\frac{\pi}{2}$. We will use the notation

$$
\beta(\alpha):=\arccos \left(\frac{4 \cos ^{2} \alpha}{\cos \alpha+1}-1\right) \quad\left(\alpha \in\left[0, \frac{\pi}{2}\right]\right) .
$$

The following estimation shows that the continuous function $\beta(\alpha)-\alpha$ is strictly increasing on the interval $\left[0, \frac{\pi}{2}\right]$ : 


$$
\begin{aligned}
\frac{d \beta(\alpha)}{d \alpha} & =\frac{4 \sin \alpha \cos \alpha(\cos \alpha+2)}{(\cos \alpha+1)^{2} \sqrt{1-\left(\frac{4 \cos ^{2} \alpha}{\cos \alpha+1}-1\right)^{2}}} \\
& =\frac{4 \sin \alpha \cos \alpha(\cos (\alpha)+2)}{(\cos \alpha+1)^{2} \sqrt{\frac{4 \cos ^{2} \alpha}{\cos \alpha+1} \sqrt{2-\frac{4 \cos ^{2} \alpha}{\cos \alpha+1}}}} \\
& =\frac{4 \sin \alpha \cos \alpha(\cos \alpha+2)}{(\cos \alpha+1) 2 \cos \alpha \sqrt{2+2 \cos \alpha-4 \cos ^{2} \alpha}} \\
& >\frac{4 \sin \alpha \cos \alpha(\cos \alpha+2)}{(\cos \alpha+1) 2 \cos \alpha \sqrt{2+2 \cos \alpha-4 \cos ^{2} \alpha}} \quad\left(0<\alpha<\frac{\pi}{2}\right) . \\
& =\frac{\cos \alpha+2}{\cos \alpha+1}=1+\frac{1}{\cos \alpha+1}>1
\end{aligned}
$$

We also know that $\beta(\alpha)-\alpha<\alpha$ holds for every $\alpha \in\left(0, \frac{\pi}{2}\right)$, and that $\beta(\alpha)-\alpha=\alpha$ if $\alpha \in\left\{0, \frac{\pi}{2}\right\}$.

Let us define a sequence $\left\{\varepsilon_{n}\right\}_{n=1}^{\infty}$ recursively as follows: $\varepsilon_{1}:=\frac{\pi}{4}, \frac{\pi}{4}<\varepsilon_{n}<\frac{\pi}{2}$ and $\beta\left(\varepsilon_{n}\right)-\varepsilon_{n}=\varepsilon_{n-1}(n \in \mathbb{N}, n \geq 2)$. This obviously gives a strictly increasing sequence which is therefore convergent, i.e. $\widehat{\varepsilon}:=\lim _{n \rightarrow \infty} \varepsilon_{n}$. By continuity, we have $\beta(\widehat{\varepsilon})-\widehat{\varepsilon}=\widehat{\varepsilon}$, from which we conclude $\widehat{\varepsilon}=\frac{\pi}{2}$.

Next, let us examine the equation $2 \pi-\beta(\alpha)-\alpha=2 \alpha$. Since the left-hand side is strictly decreasing on the interval $\left[0, \frac{\pi}{2}\right]$, and the right-hand side is strictly increasing, we conclude that there is a unique solution $\check{\varepsilon} \in\left[0, \frac{\pi}{2}\right]$. A numerical calculation gives the following two inequalities:

$$
2 \pi-\beta(1.28)-1.28>2.59>2.56=2 \cdot 1.28
$$

and

$$
2 \pi-\beta(1.29)-1.29<2.57<2.58=2 \cdot 1.29 .
$$

Therefore we infer $1.28<\check{\varepsilon}<1.29$.

We proceed with showing that if $\alpha \leq \varepsilon_{n}$ holds for some $n \in \mathbb{N}$, then $\psi$ is a $\varangle$-isometry, which will complete this case. In fact, the previous case implies this for $n=1$. Let us assume that we have already verified this for an $n \in \mathbb{N}$, and consider an $\alpha \leq \varepsilon_{n+1}$. On one hand, if $\beta(\alpha)+\alpha \leq \pi$, then by Lemma $3.6 \psi$ preservers the spherical angle $\beta(\alpha)-\alpha$ in both directions, and since $\beta(\alpha)-\alpha \leq \varepsilon_{n}$, we are done by the inductional hypothesis. On the other hand, if $\beta(\alpha)+\alpha>\pi$, then by Lemma 3.2 we have:

$$
\begin{aligned}
\varangle(x, y) \in\{\beta(\alpha)-\alpha, 2 & -\beta(\alpha)-\alpha\} \Longleftrightarrow \#\left(x^{(\alpha)} \cap y^{(\beta(\alpha))}\right)=1 \\
& \Longleftrightarrow \#\left(\psi(x)^{(\alpha)} \cap \psi(y)^{(\beta(\alpha))}\right)=1 \\
& \Longleftrightarrow \varangle(\psi(x), \psi(y)) \in\{\beta(\alpha)-\alpha, 2 \pi-\beta(\alpha)-\alpha\} .
\end{aligned}
$$

We distinguish two possibilities. First, if $\alpha \leq \check{\varepsilon}$, then we have

$\varangle(x, y)=\beta(\alpha)-\alpha \Longleftrightarrow \varangle(x, y) \in\{\beta(\alpha)-\alpha, 2 \pi-\beta(\alpha)-\alpha\}$ and $\varangle(x, y)<2 \alpha$,

thus (6) and (7) of Lemma 3.3 imply that $\psi$ preserves the spherical angle $\beta(\alpha)-\alpha$ in both directions. Since $\beta(\alpha)-\alpha \leq \varepsilon_{n}$, the map $\psi$ has to be a $\varangle$-isometry. Second, if $\alpha>\check{\varepsilon}$, then we observe that

$$
2 \pi-4 \alpha<2 \pi-4 \check{\varepsilon}<1.2<\check{\varepsilon}<\alpha .
$$


Lemma 3.2 gives the following:

$$
\varangle(x, y)=2 \pi-4 \alpha \Longleftrightarrow \#\left(x^{(2 \alpha)} \cap y^{(2 \alpha)}\right)=1 \quad\left(x, y \in S_{H}\right) .
$$

Since $\psi$ preserves the spherical angle $2 \alpha$ in both directions by Lemma 3.3, a straightforward argument shows that $\psi$ also preserves the spherical angle $2 \pi-4 \alpha$ in both directions. Therefore, by the previous possibility we infer the $\varangle$-isometriness of $\psi$.

Case 3: when $\frac{3 \pi}{4}<\alpha<\pi$. By Lemma 3.2 we have $\#\left(x^{(\alpha)} \cap y^{(\alpha)}\right)=1$ if and only if $\varangle(x, y)=2 \pi-2 \alpha$, therefore $\psi$ preserves the spherical angle $2 \pi-2 \alpha \in\left(0, \frac{\pi}{2}\right)$ in both directions, and the previous cases complete the present one.

Case 4: when $\alpha=\frac{3 \pi}{4}$. Similarly as above, we get that $\psi$ preserves the spherical angle $\frac{\pi}{2}$ in both directions. By Lemma 3.2 we have the following property:

$$
\varangle(x, y) \in\left\{\frac{\pi}{4}, \frac{3 \pi}{4}\right\} \Longleftrightarrow \varangle(\psi(x), \psi(y)) \in\left\{\frac{\pi}{4}, \frac{3 \pi}{4}\right\} \quad\left(x, y \in S_{H}\right) .
$$

But the spherical angle $\frac{3 \pi}{4}$ is preserved in both directions, whence we get that the same holds for $\frac{\pi}{4}$, and therefore, by Case $1, \psi$ is indeed a $\varangle$-isometry.

Case 5: when $\frac{\pi}{2}<\alpha<\frac{3 \pi}{4}$ and $\alpha \neq \frac{2 \pi}{3}$. We set $\epsilon_{1}:=\frac{5 \pi}{8}$ and define $\epsilon_{n}$ for $n \geq 2$ by the recursive formula $\epsilon_{n}=\pi-\frac{\epsilon_{n-1}}{2}(n \geq 2, n \in \mathbb{N})$. We observe that $\frac{5 \pi}{8} \leq \epsilon_{2 k-1}<\frac{2 \pi}{3}$ and $\frac{2 \pi}{3}<\epsilon_{2 k} \leq \frac{11 \pi}{16}$ are satisfied for all $k \in \mathbb{N}$. Moreover, one easily obtains the following explicit formula by a straightforward calculation:

$$
\epsilon_{n}=(-1)^{n-1} \frac{\epsilon_{1}}{2^{n-1}}+\sum_{j=0}^{n-2}(-1)^{j} \frac{\pi}{2^{j}} \quad(n \geq 2),
$$

whence we infer that $\lim _{n \rightarrow \infty} \epsilon_{n}=\frac{2 \pi}{3}$.

We show that if $\frac{\pi}{2}<\alpha \leq \epsilon_{n}$ and $n$ is odd, or $\epsilon_{n} \leq \alpha<\frac{3 \pi}{4}$ and $n$ is even, then $\psi$ is a $\varangle$-isometry, which will complete this case. In order to do this, we use induction. If $n=1$, then $\frac{\pi}{2}<\alpha \leq \epsilon_{1}=\frac{5 \pi}{8}$ implies that $\psi$ preserves the spherical angle $2 \pi-2 \alpha \in\left[\frac{3 \pi}{4}, \pi\right)$. From the previous cases it readily follows that $\psi$ is a $\varangle$-isometry. Let us suppose that the claim has been proven for some $n \geq 1$, and let us investigate it for $n+1$. On one hand, if $n=2 k-1$ with some $k \in \mathbb{N}$, then $\alpha \geq \epsilon_{2 k}>\frac{2 \pi}{3}$ implies that $\psi$ preserves the spherical angle $2 \pi-2 \alpha \in\left(0, \epsilon_{2 k-1}\right]$ in both directions. Thus, by the previous cases and the inductional hypothesis, either $\psi$ is a $\varangle$-isometry, or $2 \pi-2 \alpha=\frac{\pi}{2}$. But in the latter case we have $\alpha=\frac{3 \pi}{4}$ which we exclude here, therefore $\psi$ is a $\varangle$-isometry. On the other hand, if $n=2 k$ with some $k \in \mathbb{N}$, then $\frac{\pi}{2}<\alpha \leq \epsilon_{2 k+1}$ implies that $\psi$ preserves the spherical angle $2 \pi-2 \alpha \geq \epsilon_{2 k}$ in both directions. The inductional hypothesis and the previous cases together yield that $\psi$ is a $\varangle$-isometry.

Case 6: when $\alpha=\frac{2 \pi}{3}$. First, by Lemma 3.2, for every $x, y \in S_{H}$ we have

$$
\begin{aligned}
\varangle(x, y)>\frac{2 \pi}{3} & \Longleftrightarrow x^{(2 \pi / 3)} \cap y^{(2 \pi / 3)}=\emptyset \\
& \Longleftrightarrow \psi(x)^{(2 \pi / 3)} \cap \psi(y)^{(2 \pi / 3)}=\emptyset \quad \Longleftrightarrow \varangle(\psi(x), \psi(y))>\frac{2 \pi}{3} .
\end{aligned}
$$

Next, observe that for every $x \in S_{H}$ and $u \in x^{(2 \pi / 3)}$ we have $\#\left(x^{(2 \pi / 3)} \cap\right.$ $\left.u^{(2 \pi / 3)}\right)=1$, and let us denote this unique element by $\widetilde{u_{x}}$, which depends only on 
$x$ and $u$, furthermore, we also have $\widetilde{u_{x}}=\widetilde{x_{u}} \in[u, x]$. We obtain the following:

$$
\begin{aligned}
\left\{\psi\left(\widetilde{u_{x}}\right)\right\} & =\psi\left(x^{(2 \pi / 3)} \cap u^{(2 \pi / 3)}\right) \\
& =\psi(x)^{(2 \pi / 3)} \cap \psi(u)^{(2 \pi / 3)}=\left\{\widetilde{\psi(u)_{\psi(x)}}\right\} \quad\left(x \in S_{H}, u \in x^{(2 \pi / 3)}\right) .
\end{aligned}
$$

Now, we claim that $\varangle(x, y)=\pi$ holds if and only if $\varangle(x, y)>\frac{2 \pi}{3}$ and for every $u \in x^{(2 \pi / 3)}$ and $z \in y^{(2 \pi / 3)} \cap u^{(2 \pi / 3)}$ we have $\varangle\left(\widetilde{u_{x}}, \widetilde{z_{y}}\right)=\frac{2 \pi}{3}$. For the necessity part, suppose that $\varangle(x, y)=\pi$, and consider the following isometry:

$$
T: H \rightarrow H, \quad T(\lambda x+h)=\lambda x-h \quad(h \in H, h \perp x) .
$$

Since we have $\widetilde{u_{x}}=T u$ and $\widetilde{z_{y}}=T z$, therefore indeed, $\varangle\left(\widetilde{u_{x}}, \widetilde{z_{y}}\right)=\varangle(u, z)=\frac{2 \pi}{3}$.

We proceed with the sufficiency part. Assume that $\frac{2 \pi}{3}<\varangle(x, y)<\pi$ holds. We shall find a vector $u \in x^{(2 \pi / 3)}$ such that for every $z \in y^{(2 \pi / 3)} \cap u^{(2 \pi / 3)}$ we have $\varangle\left(\widetilde{u_{x}}, \widetilde{z_{y}}\right) \neq \frac{2 \pi}{3}$. Set $\gamma:=\varangle(x, y)$ and choose an orthonormal system $\left\{e_{1}, e_{2}\right\}$ such that $x=e_{1}$ and $y=\cos \gamma \cdot e_{1}+\sin \gamma \cdot e_{2}$. We define $y^{\prime}:=\sin \gamma \cdot e_{1}-\cos \gamma \cdot e_{2}$ (which is orthogonal to $y$ ) and fix the vector $u:=-\frac{1}{2} \cdot e_{1}-\frac{\sqrt{3}}{2} \cdot e_{2} \in x^{(2 \pi / 3)}$. We have $\widetilde{u_{x}}=-\frac{1}{2} \cdot e_{1}+\frac{\sqrt{3}}{2} \cdot e_{2}$. A straightforward calculation gives that for any $z \in y^{(2 \pi / 3)}$ there exists a $\delta \in[0,2 \pi)$ and an $e_{3} \in S_{H}, e_{3} \perp e_{j}(j=1,2)$ such that

$$
z=-\frac{1}{2} \cdot y+\frac{\sqrt{3}}{2} \cdot\left(\cos \delta \cdot y^{\prime}+\sin \delta \cdot e_{3}\right)
$$

and then

$$
\widetilde{z_{y}}=-\frac{1}{2} \cdot y-\frac{\sqrt{3}}{2} \cdot\left(\cos \delta \cdot y^{\prime}+\sin \delta \cdot e_{3}\right) .
$$

Now, we have $z \in y^{(2 \pi / 3)} \cap u^{(2 \pi / 3)}$ if and only if the following equation is satisfied:

$$
\langle u, z\rangle=\frac{1}{4} \cos \gamma+\frac{\sqrt{3}}{4} \sin \gamma-\frac{\sqrt{3}}{4} \sin \gamma \cos \delta+\frac{3}{4} \cos \gamma \cos \delta=-\frac{1}{2} .
$$

Assume we also have $\varangle\left(\widetilde{u_{x}}, \widetilde{z_{y}}\right)=\frac{2 \pi}{3}$. Note that this happens exactly when

$$
\left\langle\widetilde{u_{x}}, \widetilde{z_{y}}\right\rangle=\frac{1}{4} \cos \gamma-\frac{\sqrt{3}}{4} \sin \gamma+\frac{\sqrt{3}}{4} \sin \gamma \cos \delta+\frac{3}{4} \cos \gamma \cos \delta=-\frac{1}{2} .
$$

Subtracting (11) from (12) gives $\sin \gamma=\sin \gamma \cos \delta$, which implies $\cos \delta=1$. Then by (11) we get $\frac{2 \pi}{3}=\gamma$, a contradiction, therefore our claim is verified.

Finally, by the observations made above we infer the following equivalence-chain:

$$
\begin{aligned}
\varangle(x, y)=\pi & \Longleftrightarrow\left\{\begin{array}{c}
\varangle(x, y)>\frac{2 \pi}{3} \text { and } \varangle\left(\widetilde{u_{x}}, \widetilde{z_{y}}\right)=\frac{2 \pi}{3} \\
\text { for every } u \in x^{(2 \pi / 3)}, z \in y^{(2 \pi / 3)} \cap u^{(2 \pi / 3)}
\end{array}\right. \\
& \Longleftrightarrow\left\{\begin{array}{c}
\varangle(\psi(x), \psi(y))>\frac{2 \pi}{3} \text { and } \varangle\left(\widetilde{\left.\psi(u)_{\psi(x)}\right)}, \widetilde{(z)_{\psi(y)}}\right)=\frac{2 \pi}{3} \\
\text { for every } \psi(u) \in \psi(x)^{(2 \pi / 3)}, \psi(z) \in \psi(y)^{(2 \pi / 3)} \cap \psi(u)^{(2 \pi / 3)}
\end{array}\right. \\
& \Longleftrightarrow\left\{\begin{array}{c}
\varangle(\psi(x), \psi(y))>\frac{2 \pi}{3} \text { and } \varangle\left(\widetilde{w_{\psi(x)}}, \widetilde{s_{\psi(y)}}\right)=\frac{2 \pi}{3} \\
\text { for every } w \in \psi(x)^{(2 \pi / 3)}, s \in \psi(y)^{(2 \pi / 3)} \cap w^{(2 \pi / 3)}
\end{array}\right. \\
& \Longleftrightarrow \varangle(\psi(x), \psi(y))=\pi .
\end{aligned}
$$

Since $\psi$ preserves the spherical angles $\frac{2 \pi}{3}$ and $\pi$ in both directions, one easily sees that it also preserves the spherical angle $\frac{\pi}{3}$ in both directions, hence Case 2 completes the proof. 


\section{Symmetries of PROJECtive SPACES}

The aim of this section is to prove our main results on transformations of projective spaces namely, Theorems 1, 3 and 4 . We begin with the verification of Theorem 3 which is a direct consequence of Theorem 2 .

Proof of Theorem 3. By Bloch's representation (see e.g. [31]), elements of $P\left(\mathbb{C}^{2}\right)$ can be represented as points on the unit sphere $S_{\mathbb{R}^{3}}$ of $\mathbb{R}^{3}$ in the following way:

$$
\rho: P\left(\mathbb{C}^{2}\right) \rightarrow S_{\mathbb{R}^{3}}, \quad \rho\left(\left[\left(\cos \theta, e^{i \nu} \sin \theta\right)\right]\right)=(\sin 2 \theta \cos \nu, \sin 2 \theta \sin \nu, \cos 2 \theta),
$$

where $0 \leq \theta \leq \frac{\pi}{2}, 0 \leq \nu<2 \pi$. Furthermore, we have

$$
\varangle(\rho([u]), \rho([v]))=2 \cdot \measuredangle([u],[v]) \quad([u],[v] \in P(H)) .
$$

Clearly, $\rho \circ \phi \circ \rho^{-1}: S_{\mathbb{R}^{3}} \rightarrow S_{\mathbb{R}^{3}}$ preserves the spherical angle $2 \alpha$ in both directions. On one hand, if $\alpha \neq \frac{\pi}{4}$, then by Theorem 2 the map $\rho \circ \phi \circ \rho^{-1}$ is a $\varangle$-isometry, and thus $\phi$ is a Wigner symmetry. On the other hand, if $\alpha=\frac{\pi}{4}$, then from Theorem 2 we get that there is a $\varangle$-isometry $\psi: S_{\mathbb{R}^{3}} \rightarrow S_{\mathbb{R}^{3}}$ such that $\rho \circ \phi \circ \rho^{-1}(x) \in\{\psi(x),-\psi(x)\}$ holds for every $x \in S_{\mathbb{R}^{3}}$, whence we infer $\phi([v]) \in\left\{\rho^{-1} \circ \psi \circ \rho([v]), \rho^{-1} \circ \psi \circ \rho([v])^{\perp}\right\}$ for all $[v] \in P\left(\mathbb{C}^{2}\right)$, which completes the proof.

We proceed with verifying some lemmas. For any $[v] \subset P(H)$ and $0<\alpha \leq \frac{\pi}{2}$ we will use the following notation:

$$
[v]^{\alpha}:=\{[u] \in P(H): \measuredangle([u],[v])=\alpha\} .
$$

We will denote by $\mathcal{C}$ the set of those numbers in $\mathbb{K} \in\{\mathbb{C}, \mathbb{R}\}$ which have unit modulus. Furthermore, if $[v],[w] \in P(H)$, then the symbol

$$
P_{[v],[w]}:=\{[u] \in P(H): \exists \lambda, \mu \in \mathbb{K} \text { such that } u=\lambda \cdot v+\mu \cdot w \neq 0\}
$$

will stand for the projective line spanned by $[v]$ and $[w]$.

Lemma 4.1. Let $H$ be a real or complex inner product space with $\operatorname{dim} H \geq 3$. Assume that $0<\alpha \leq \beta \leq \frac{\pi}{2}$, $v, w \in H$ with $\|v\|=\|w\|=1$ and set $\gamma:=$ $\measuredangle([v],[w]) \neq 0$. Then we have

$$
\begin{aligned}
& {[v]^{\alpha} \cap[w]^{\beta}=\emptyset } \Longleftrightarrow \gamma<\beta-\alpha \text { or } \gamma>\alpha+\beta, \\
& \#\left([v]^{\alpha} \cap[w]^{\beta}\right)=1 \Longleftrightarrow\left\{\begin{array}{r}
\text { either } \gamma=\beta-\alpha, \text { or } \gamma=\alpha+\beta<\frac{\pi}{2}, \\
\text { or } \operatorname{dim} H=3 \text { and } \alpha=\beta=\frac{\pi}{2} .
\end{array}\right.
\end{aligned}
$$

Moreover, if $\gamma=\frac{\pi}{2}=\alpha+\beta$, then $[v]^{\alpha} \cap[w]^{\beta}=\{[\cos \alpha \cdot v+\lambda \sin \alpha \cdot w]: \lambda \in \mathcal{C}\}$.

Proof. If $\alpha=\frac{\pi}{2}$, then we also have $\beta=\frac{\pi}{2}$, and clearly, $\#\left([v]^{\pi / 2} \cap[w]^{\pi / 2}\right)=1$ holds if and only if $\operatorname{dim} H=3$. Therefore from now on we may assume that $0<\alpha<\frac{\pi}{2}$. By the triangle inequality, (14) is obvious, therefore we will further assume throughout the proof that $\beta-\alpha \leq \gamma \leq \alpha+\beta$ is fulfilled. We fix an orthonormal system $\left\{e_{1}, e_{2}\right\}$ such that $[v]=\left[e_{1}\right]$ and $[w]=\left[\cos \gamma \cdot e_{1}+\sin \gamma \cdot e_{2}\right]$. If $[u] \in[v]^{\alpha}$, then

$$
[u]=\left[\cos \alpha \cdot e_{1}+\lambda \sin \alpha \cos \delta \cdot e_{2}+\mu \sin \alpha \sin \delta \cdot e_{3}\right]
$$

holds with some $\delta \in\left[0, \frac{\pi}{2}\right],|\lambda|=|\mu|=1$, and $e_{3} \in H$ such that $\left\|e_{3}\right\|=1, e_{3} \perp e_{j}$ $(j=1,2)$. Of course, the three coordinates in (16) are all non-zero if and only if $0<\delta<\frac{\pi}{2}$. Note that we have $[u] \in[v]^{\alpha} \cap[w]^{\beta}$ if and only if

$$
\cos \beta=|\cos \alpha \cos \gamma+\lambda \sin \alpha \sin \gamma \cos \delta| \text {. }
$$

Now, observe that if any $(\lambda, \delta) \in \mathcal{C} \times\left(0, \frac{\pi}{2}\right]$ solves $(17)$, then choosing $\mu=-1$ and 1 in (16) gives us two different elements of $[v]^{\alpha} \cap[w]^{\beta}$. Therefore having 
$\#\left([v]^{\alpha} \cap[w]^{\beta}\right)=1$ implies $\delta=0$ for every solution $(\lambda, \delta)$ of (17). In particular, we have $[u] \in P_{[v],[w]}$ as well in that case.

Clearly, the following map is injective on $\mathcal{C} \times\left[0, \frac{\pi}{2}\right)$ :

$$
\vartheta: \mathcal{C} \times\left[0, \frac{\pi}{2}\right] \rightarrow \mathbb{K}, \quad(\lambda, \delta) \mapsto \cos \alpha \cos \gamma+\lambda \sin \alpha \sin \gamma \cos \delta
$$

Furthermore, we have $\vartheta\left(\mathcal{C} \times\left[0, \frac{\pi}{2}\right)\right)=\{z \in \mathbb{K}: 0<|z-\cos \alpha \cos \gamma| \leq \sin \alpha \sin \gamma\}$ and $\vartheta\left(\mathcal{C} \times\left\{\frac{\pi}{2}\right\}\right)=\{\cos \alpha \cos \gamma\}$. Therefore $\#\left([v]^{\alpha} \cap[w]^{\beta}\right)>0$ holds if and only if $\cos (\alpha+\gamma) \leq \cos \beta \leq \cos (\alpha-\gamma)$ does, which is equivalent to our assumption, i.e. $\beta-\alpha \leq \gamma \leq \alpha+\beta$.

Now, the following equivalence-chain is straightforward from the observations made above, and it verifies (15):

$$
\begin{aligned}
& \#\left([v]^{\alpha} \cap[w]^{\beta}\right)=1 \Longleftrightarrow(\lambda, \delta)=(-1,0) \text { or }(1,0) \text { is a unique solution of }(17) \\
& \Longleftrightarrow\left\{\begin{array}{c}
(\lambda, \delta)=(1,0) \text { solves }(17) \text { and } \cos \alpha \cos \gamma \neq 0, \text { or } \\
(\lambda, \delta)=(-1,0) \text { solves }(17) \text { and } \cos \alpha \cos \gamma \geq \sin \alpha \sin \gamma
\end{array}\right. \\
& \Longleftrightarrow\left\{\begin{array}{c}
(\lambda, \delta)=(1,0) \text { is a solution of }(17) \text { and } \gamma \neq \frac{\pi}{2}, \text { or } \\
(\lambda, \delta)=(-1,0) \text { is a solution of }(17) \text { and } \alpha+\gamma \leq \frac{\pi}{2}
\end{array}\right. \\
& \Longleftrightarrow \cos \beta=\cos (\alpha-\gamma) \text { and } \gamma \neq \frac{\pi}{2} \text {, or } \cos \beta=\cos (\alpha+\gamma) \text { and } \alpha+\gamma \leq \frac{\pi}{2} \\
& \Longleftrightarrow \beta=\alpha-\gamma \text { and } \gamma \neq \frac{\pi}{2}, \text { or } \beta=\gamma-\alpha \text { and } \gamma \neq \frac{\pi}{2}, \text { or } \beta=\alpha+\gamma \\
& \Longleftrightarrow \gamma=\alpha-\beta, \text { or } \gamma=\alpha+\beta<\frac{\pi}{2}, \text { or } \gamma=\beta-\alpha \\
& \Longleftrightarrow \gamma=\alpha+\beta<\frac{\pi}{2} \text { or } \gamma=\beta-\alpha .
\end{aligned}
$$

Finally, if $\gamma=\frac{\pi}{2}=\alpha+\beta$, then (17) becomes $\sin \alpha=\cos \beta=\sin \alpha \cos \delta$, therefore $\delta=0$, and by $(16)$ we get

$[v]^{\alpha} \cap[w]^{\beta} \subseteq[v]^{\alpha} \subseteq\left\{\left[\cos \alpha \cdot e_{1}+\lambda \sin \alpha \cdot e_{2}\right]: \lambda \in \mathcal{C}\right\}=\{[\cos \alpha \cdot v+\lambda \sin \alpha \cdot w]: \lambda \in \mathcal{C}\}$. But obviously, $\{[\cos \alpha \cdot v+\lambda \sin \alpha \cdot w]: \lambda \in \mathcal{C}\} \subseteq[v]^{\alpha} \cap[w]^{\beta}$ is also fulfilled.

The following three lemmas are consequences of Lemma 4.1 and are proven in a similar way as the lemmas in Section 3.

Lemma 4.2. Let $H$ be a real or complex inner product space with $\operatorname{dim} H \geq 3$, and $\phi: P(H) \rightarrow P(H)$ be a bijection. If $\phi$ preserves the quantum angle $\alpha \in\left(0, \frac{\pi}{4}\right)$ in both directions, then $\phi$ shares the following property:

$$
\measuredangle([v],[w]) \leq j \alpha \Longleftrightarrow \measuredangle(\phi([v]), \phi([w])) \leq j \alpha \quad\left([v],[w] \in P(H), 2 \leq j<\frac{\pi}{2 \alpha}\right) .
$$

Proof. Observe that we have $\measuredangle([v],[w]) \leq j \alpha\left(j \in \mathbb{N}, 2 \leq j<\frac{\pi}{2 \alpha}\right)$ if and only if there exists a $(j-1)$-element sequence $\left\{\left[u_{l}\right]\right\}_{l=1}^{j-1} \subset P(H)$ with $\measuredangle\left([v],\left[u_{1}\right]\right)=$ $\measuredangle\left(\left[u_{l}\right],\left[u_{l+1}\right]\right)=\measuredangle\left(\left[u_{j-1}\right],[w]\right)=\alpha(l \in \mathbb{N}, 1 \leq l \leq j-2)$. Indeed, one direction is immediate from the triangle inequality. Concerning the other one, by Bloch's representation we infer the existence of such a $(j-1)$-element sequence in $P_{[v],[w]}$. Therefore, using the same technique as in Lemma 3.3, (18) is yielded.

Lemma 4.3. Let $H$ be a real or complex inner product space with $\operatorname{dim} H \geq 3$, $0<\alpha<\beta<\frac{\pi}{2}$, and $\phi: P(H) \rightarrow P(H)$ be a bijection. If $\phi$ preserves the quantum angles $\alpha$ and $\beta$ in both directions, then

(i) $\phi$ also preserves the quantum angles $\beta-\alpha$ and $\alpha+\beta$ in both directions if $\alpha+\beta<\frac{\pi}{2}$

(ii) $\phi$ also preserves the quantum angle $\beta-\alpha$ in both directions if $\alpha+\beta \geq \frac{\pi}{2}$. 
Proof. (i): Applying Lemma 4.1 gives the following for every $[v],[w] \in P(H)$ :

$$
\measuredangle([v],[w]) \in\{\beta-\alpha, \alpha+\beta\} \Longleftrightarrow \measuredangle(\phi([v]), \phi([w])) \in\{\beta-\alpha, \alpha+\beta\} .
$$

Let us observer that there exists a $j \in \mathbb{N}, 2 \leq j<\frac{\pi}{2 \alpha}$ with $\beta-\alpha<j \alpha<\alpha+\beta$. A technique similar to the one used in Lemma 3.6 completes the proof of this case.

(ii): A similar, but easier argument verifies this part.

Lemma 4.4. Let $H$ be a real or complex inner product space with $\operatorname{dim} H \geq 3$, and $\phi: P(H) \rightarrow P(H)$ be a bijection. If $\phi$ preserves the quantum angle $\alpha \in\left(0, \frac{\pi}{4}\right)$ in both directions, then $\phi$ shares the following property:

$$
\measuredangle([v],[w])=j \alpha \Longleftrightarrow \measuredangle(\phi([v]), \phi([w]))=j \alpha \quad\left([v],[w] \in P(H), 2 \leq j<\frac{\pi}{2 \alpha}\right) .
$$

Proof. For $j=2$, this is straightforward from Lemma 4.1. For $j>2$, the statement can be verified using a simple recursion and (i) of Lemma 4.3.

The next lemma can be verified along the same lines as Lemma 3.5.

Lemma 4.5. Let $H$ be a real or complex inner product space with $\operatorname{dim} H \geq 3$, and $\phi: P(H) \rightarrow P(H)$ be a bijection. If there is a sequence of positive quantum angles $\left\{\alpha_{n}\right\}_{n=1}^{\infty}$ such that $\lim _{n \rightarrow \infty} \alpha_{n}=0$ and $\phi$ preserves these quantum angles in both directions, then $\phi$ is a Wigner symmetry.

If $[v] \in P(H)$, then the symbol $H \ominus[v]$ will denote the set of those vectors in $H$ which are orthogonal to $v$. This is a linear subspace, thus it can be considered as an inner product space with the restricted inner product.

Now, we are in the position to verify Theorem 1 by applying Theorem 2 .

Proof of Theorem 1. During our proof we will distinguish six different cases. In the first three of them we will deal with the possibility when $H$ is of dimension at least four, and in the last three ones we will handle the three-dimensional case.

Case 1: when $\operatorname{dim} H \geq 4$ and $0<\alpha<\frac{\pi}{3}$. Let $v \in S_{H}$ be arbitrary and $w \in S_{H}$ such that $\phi([v])=[w]$ holds. Then we have $\phi\left([v]^{\alpha}\right)=[w]^{\alpha}$. We consider a bijective isometry $R: H \rightarrow H$ such that $R w=v$, and define the transformation $\widetilde{\phi}: P(H) \rightarrow P(H), \widetilde{\phi}([u])=R(\phi([u]))$ which is also bijective and preserves the quantum angle $\alpha$ in both directions. In addition, we have $\widetilde{\phi}([v])=[v]$, whence $\widetilde{\phi}\left([v]^{\alpha}\right)=[v]^{\alpha}$ follows as well. It is straightforward that

$$
[v]^{\alpha}=\left\{[\cos \alpha \cdot v+\sin \alpha \cdot u]: u \in S_{H \ominus[v]}\right\}
$$

where $\operatorname{dim}(H \ominus[v]) \geq 3$. Therefore there exists a bijective map $\psi: S_{H \ominus[v]} \rightarrow S_{H \ominus[v]}$ which induces the restricted transformation $\left.\widetilde{\phi}\right|_{[v]^{\alpha}}$, i.e. we have

$$
\widetilde{\phi}([\cos \alpha \cdot v+\sin \alpha \cdot u])=[\cos \alpha \cdot v+\sin \alpha \cdot \psi(u)] \quad\left(u \in S_{H \ominus[v]}\right) .
$$

Our aim is to show that $\psi$ is a $\varangle$-isometry. We calculate the following for every $u_{1}, u_{2} \in S_{H \ominus[v]}$ :

$\measuredangle\left(\left[\cos \alpha \cdot v+\sin \alpha \cdot u_{1}\right],\left[\cos \alpha \cdot v+\sin \alpha \cdot u_{2}\right]\right)=\arccos \left(\left|\cos ^{2} \alpha+\left\langle u_{1}, u_{2}\right\rangle \sin ^{2} \alpha\right|\right)$.

If $u_{1}$ and $u_{2}$ run through $S_{H \ominus[v]}$, then $\cos ^{2} \alpha+\left\langle u_{1}, u_{2}\right\rangle \sin ^{2} \alpha$ runs through the interval $[\cos 2 \alpha, 1]$, and thus the quantum angle in $(21)$ runs through $\left[0, \min \left(\frac{\pi}{2}, 2 \alpha\right)\right]$. 
The inequality $-\cos \alpha<\cos 2 \alpha$ implies that

$$
\measuredangle\left(\left[\cos \alpha \cdot v+\sin \alpha \cdot u_{1}\right],\left[\cos \alpha \cdot v+\sin \alpha \cdot u_{2}\right]\right)=\alpha
$$

holds exactly when

$$
\varangle\left(u_{1}, u_{2}\right)=\arccos \left(\frac{\cos \alpha}{1+\cos \alpha}\right) \in\left(\frac{\pi}{3}, \arccos \frac{1}{3}\right) \subset\left(\frac{\pi}{3}, \frac{\pi}{2}\right) .
$$

Since $\widetilde{\phi}$ preserves the quantum angle $\alpha$ in both directions, it is apparent that $\psi$ preserves the spherical angle $\arccos \left(\frac{\cos \alpha}{1+\cos \alpha}\right)$ in both directions. By Theorem 2 we obtain that $\psi$ is a $\varangle$-isometry. Therefore if $\left[w_{1}\right],\left[w_{2}\right] \in[v]^{\alpha}$, then we have $\measuredangle\left(\left[w_{1}\right],\left[w_{2}\right]\right)=\measuredangle\left(\widetilde{\phi}\left(\left[w_{1}\right]\right), \widetilde{\phi}\left(\left[w_{2}\right]\right)\right)$, and thus we infer

$$
\measuredangle\left(\phi\left(\left[w_{1}\right]\right), \phi\left(\left[w_{2}\right]\right)\right)=\measuredangle\left(\left[w_{1}\right],\left[w_{2}\right]\right) \quad\left(\left[w_{1}\right],\left[w_{2}\right] \in[v]^{\alpha}\right) .
$$

Since this holds for every line $[v]$, our map $\phi$ preserves every quantum angle from the interval $\left(0, \max \left(2 \alpha, \frac{\pi}{2}\right)\right]$, hence $\phi$ has to be a Wigner symmetry by Lemma 4.5 .

Case 2: when $\operatorname{dim} H \geq 4$ and $\frac{\pi}{3}<\alpha<\frac{\pi}{2}$. In this case we have

$$
\measuredangle\left(\left[\cos \alpha \cdot v+\sin \alpha \cdot u_{1}\right],\left[\cos \alpha \cdot v+\sin \alpha \cdot u_{2}\right]\right)=\alpha
$$

if and only if

$$
\varangle\left(u_{1}, u_{2}\right) \in\left\{\beta_{1}, \beta_{2}\right\}
$$

where

$$
\beta_{1}:=\arccos \left(1-\frac{1}{1+\cos \alpha}\right) \in\left(\arccos \frac{1}{3}, \frac{\pi}{2}\right)
$$

and

$$
\beta_{2}:=\arccos \left(1-\frac{1}{1-\cos \alpha}\right) \in\left(\frac{\pi}{2}, \pi\right) .
$$

Let us observe that, similarly as in the previous case, if one was able to show that every bijection $\psi: S_{H \ominus[v]} \rightarrow S_{H \ominus[v]}$ which satisfies

$$
\varangle(x, y) \in\left\{\beta_{1}, \beta_{2}\right\} \Longleftrightarrow \varangle(\psi(x), \psi(y)) \in\left\{\beta_{1}, \beta_{2}\right\}
$$

is a $\varangle$-isometry, then one could conclude that $\phi$ is a Wigner symmetry. That is exactly what will be done here.

Let $x, y \in S_{H \ominus[v]}$ be two unit vectors with $\gamma:=\varangle(x, y) \neq 0$. Obviously, we have

$$
\begin{aligned}
\#\left(\left(x^{\left(\beta_{1}\right)} \cup x^{\left(\beta_{2}\right)}\right)\right. & \left.\cap\left(y^{\left(\beta_{1}\right)} \cup y^{\left(\beta_{2}\right)}\right)\right) \\
& =\#\left(x^{\left(\beta_{1}\right)} \cap y^{\left(\beta_{1}\right)}\right)+2 \cdot \#\left(x^{\left(\beta_{1}\right)} \cap y^{\left(\beta_{2}\right)}\right)+\#\left(x^{\left(\beta_{2}\right)} \cap y^{\left(\beta_{2}\right)}\right) .
\end{aligned}
$$

The core idea here is to examine this cardinality. Let us observe that $\cos \beta_{1}<$ $-\cos \beta_{2}$, which implies $\beta_{1}+\beta_{2}>\pi$. By Lemma 3.2 we obtain the following equivalences:

$$
\begin{gathered}
\#\left(x^{\left(\beta_{1}\right)} \cap y^{\left(\beta_{1}\right)}\right)=1 \Longleftrightarrow \gamma=2 \beta_{1}, \\
x^{\left(\beta_{1}\right)} \cap y^{\left(\beta_{1}\right)}=\emptyset \Longleftrightarrow \gamma>2 \beta_{1}, \\
\#\left(x^{\left(\beta_{1}\right)} \cap y^{\left(\beta_{2}\right)}\right)=1 \Longleftrightarrow \gamma \in\left\{2 \pi-\beta_{1}-\beta_{2}, \beta_{2}-\beta_{1}\right\}, \\
x^{\left(\beta_{1}\right)} \cap y^{\left(\beta_{2}\right)}=\emptyset \Longleftrightarrow \gamma>2 \pi-\beta_{1}-\beta_{2} \text { or } \gamma<\beta_{2}-\beta_{1}, \\
\#\left(x^{\left(\beta_{2}\right)} \cap y^{\left(\beta_{2}\right)}\right)=1 \Longleftrightarrow \gamma=2 \pi-2 \beta_{2},
\end{gathered}
$$


and

$$
x^{\left(\beta_{2}\right)} \cap y^{\left(\beta_{2}\right)}=\emptyset \Longleftrightarrow \gamma>2 \pi-2 \beta_{2} .
$$

Notice that if $\operatorname{dim}(H \ominus[v])=3$, then $\#\left(x^{\left(\beta_{j}\right)} \cap y^{\left(\beta_{l}\right)}\right) \in\{0,1,2\}$ holds, and if $\operatorname{dim}(H \ominus[v]) \geq 4$, then always we have $\#\left(x^{\left(\beta_{j}\right)} \cap y^{\left(\beta_{l}\right)}\right) \in\{0,1, \infty\}(j, l \in\{1,2\})$.

Our next step is to compare the following four quantities: $2 \beta_{1}, 2 \pi-\beta_{1}-\beta_{2}, \beta_{2}-$ $\beta_{1}, 2 \pi-2 \beta_{2}$. Since we have $\frac{\pi}{3}<\beta_{1}$, we get $2 \beta_{1}>\beta_{2}-\beta_{1}$. By similar observations, we obtain the following inequalities: $2 \beta_{1}>2 \pi-2 \beta_{2}, 2 \pi-\beta_{1}-\beta_{2}>\beta_{2}-\beta_{1}$, $2 \pi-\beta_{1}-\beta_{2}>2 \pi-2 \beta_{2}$; and the following relations: $2 \beta_{1}, 2 \pi-\beta_{1}-\beta_{2}, \beta_{2}-\beta_{1}, 2 \pi-$ $2 \beta_{2} \in(0, \pi)$.

Now, let us solve the equation $2 \beta_{1}=2 \pi-\beta_{1}-\beta_{2}$, which is equivalent to $3 \beta_{1}=2 \pi-\beta_{2}$. Since both sides are in the interval $\left(\pi, \frac{3 \pi}{2}\right)$, this holds if and only if

$$
\begin{aligned}
1-\frac{1}{1-\cos \alpha} & =\cos \beta_{2}=\cos \left(3 \beta_{1}\right)=4 \cos ^{3}\left(\beta_{1}\right)-3 \cos \left(\beta_{1}\right) \\
& =4\left(1-\frac{1}{1+\cos \alpha}\right)^{3}-3\left(1-\frac{1}{1+\cos \alpha}\right) .
\end{aligned}
$$

By a straightforward calculation we get that this holds exactly when $\cos \alpha \in$ $\left\{-\frac{1}{\sqrt{5}}, 0, \frac{1}{\sqrt{5}}\right\}$. Clearly, the first two possibilities cannot occur, therefore we conclude the only solution $\alpha=\arccos \left(\frac{1}{\sqrt{5}}\right)$.

Next, we examine the equation $\beta_{2}-\beta_{1}=2 \pi-2 \beta_{2}$, which is the same as $3 \beta_{2}=$ $2 \pi+\beta_{1}$. This implies

$$
\begin{aligned}
1-\frac{1}{1+\cos \alpha} & =\cos \beta_{1}=\cos \left(3 \beta_{2}\right)=4 \cos ^{3}\left(\beta_{2}\right)-3 \cos \left(\beta_{2}\right) \\
& =4\left(1-\frac{1}{1-\cos \alpha}\right)^{3}-3\left(1-\frac{1}{1-\cos \alpha}\right) .
\end{aligned}
$$

Again, quite straightforwardly, the unique solution is $\alpha=\arccos \left(\frac{1}{\sqrt{5}}\right)$.

By the above observations we have at most the following six possibilities concerning the order of the four quantities $2 \beta_{1}, 2 \pi-\beta_{1}-\beta_{2}, \beta_{2}-\beta_{1}, 2 \pi-2 \beta_{2}$ :

1. $2 \beta_{1}=2 \pi-\beta_{1}-\beta_{2}>\beta_{2}-\beta_{1}=2 \pi-2 \beta_{2}$;

2. $2 \beta_{1}>2 \pi-\beta_{1}-\beta_{2}>\beta_{2}-\beta_{1}>2 \pi-2 \beta_{2}$;

3. $\operatorname{dim} H=4$ and $2 \pi-\beta_{1}-\beta_{2}>2 \beta_{1}>\beta_{2}-\beta_{1}>2 \pi-2 \beta_{2}$;

4. $\operatorname{dim} H \geq 5$ and $2 \pi-\beta_{1}-\beta_{2}>2 \beta_{1}>\beta_{2}-\beta_{1}>2 \pi-2 \beta_{2}$;

5. $2 \beta_{1}>2 \pi-\beta_{1}-\beta_{2}>2 \pi-2 \beta_{2}>\beta_{2}-\beta_{1}$;

6. $2 \pi-\beta_{1}-\beta_{2}>2 \beta_{1}>2 \pi-2 \beta_{2}>\beta_{2}-\beta_{1}$.

Using (24)-(29), in each of the above six cases we get the following equivalences:

1. $\#\left(\left(x^{\left(\beta_{1}\right)} \cup x^{\left(\beta_{2}\right)}\right) \cap\left(y^{\left(\beta_{1}\right)} \cup y^{\left(\beta_{2}\right)}\right)\right)=3 \Longleftrightarrow \gamma=2 \beta_{1}=2 \pi-\beta_{1}-\beta_{2}$;

2. \# $\left(\left(x^{\left(\beta_{1}\right)} \cup x^{\left(\beta_{2}\right)}\right) \cap\left(y^{\left(\beta_{1}\right)} \cup y^{\left(\beta_{2}\right)}\right)\right)=1 \Longleftrightarrow \gamma=2 \beta_{1}$;

3. \# $\left(\left(x^{\left(\beta_{1}\right)} \cup x^{\left(\beta_{2}\right)}\right) \cap\left(y^{\left(\beta_{1}\right)} \cup y^{\left(\beta_{2}\right)}\right)\right)=5 \Longleftrightarrow \gamma=2 \beta_{1}$;

4. \# $\left(\left(x^{\left(\beta_{1}\right)} \cup x^{\left(\beta_{2}\right)}\right) \cap\left(y^{\left(\beta_{1}\right)} \cup y^{\left(\beta_{2}\right)}\right)\right)=2 \Longleftrightarrow \gamma=2 \pi-\beta_{1}-\beta_{2}$;

5. \# $\left(\left(x^{\left(\beta_{1}\right)} \cup x^{\left(\beta_{2}\right)}\right) \cap\left(y^{\left(\beta_{1}\right)} \cup y^{\left(\beta_{2}\right)}\right)\right)=1 \Longleftrightarrow \gamma=2 \beta_{1}$;

6. \# $\left(\left(x^{\left(\beta_{1}\right)} \cup x^{\left(\beta_{2}\right)}\right) \cap\left(y^{\left(\beta_{1}\right)} \cup y^{\left(\beta_{2}\right)}\right)\right)=2 \Longleftrightarrow \gamma=2 \pi-\beta_{1}-\beta_{2}$.

Here we only give the verification of the second point above, as the others are very similar. For this let us consider the following table: 


\begin{tabular}{|c|c|c|c|}
\hline & $\#\left(x^{\left(\beta_{1}\right)} \cap y^{\left(\beta_{1}\right)}\right)$ & $\#\left(x^{\left(\beta_{1}\right)} \cap y^{\left(\beta_{2}\right)}\right)$ & $\#\left(x^{\left(\beta_{2}\right)} \cap y^{\left(\beta_{2}\right)}\right)$ \\
\hline $0<\gamma<2 \pi-2 \beta_{2}$ & $2 \leq$ & 0 & $2 \leq$ \\
\hline$\gamma=2 \pi-2 \beta_{2}$ & $2 \leq$ & 0 & 1 \\
\hline $2 \pi-2 \beta_{2}<\gamma<\beta_{2}-\beta_{1}$ & $2 \leq$ & 0 & 0 \\
\hline$\gamma=\beta_{2}-\beta_{1}$ & $2 \leq$ & 1 & 0 \\
\hline$\beta_{2}-\beta_{1}<\gamma<2 \pi-\beta_{1}-\beta_{2}$ & $2 \leq$ & $2 \leq$ & 0 \\
\hline$\gamma=2 \pi-\beta_{1}-\beta_{2}$ & $2 \leq$ & 1 & 0 \\
\hline $2 \pi-\beta_{1}-\beta_{2}<\gamma<2 \beta_{1}$ & $2 \leq$ & 0 & 0 \\
\hline$\gamma=2 \beta_{1}$ & 1 & 0 & 0 \\
\hline $2 \beta_{1}<\gamma \leq \pi$ & 0 & 0 & 0 \\
\hline
\end{tabular}

Clearly, (23) gives us the desired conclusion.

Finally, in each of the cases above we conclude that $\psi$ preserves the spherical angle $2 \beta_{1}$ or $2 \pi-\beta_{1}-\beta_{2}$ in both directions. The only thing which needs to be shown is that they cannot be $\frac{\pi}{2}$, which is obvious for the first one. Concerning the second one, we know that $\beta_{1}<\frac{\pi}{2}$ and $\beta_{2}<\pi$, thus $2 \pi-\beta_{1}-\beta_{2}>\frac{\pi}{2}$.

Case 3: when $\operatorname{dim} H \geq 4$ and $\alpha=\frac{\pi}{3}$. Similarly as in the previous case, we only need to show that any bijective map $\psi: S_{H \ominus[v]} \rightarrow S_{H \ominus[v]}$ which satisfies

$$
\varangle(x, y) \in\left\{\arccos \left(\frac{1}{3}\right), \pi\right\} \Longleftrightarrow \varangle(\psi(x), \psi(y)) \in\left\{\arccos \left(\frac{1}{3}\right), \pi\right\}
$$

is a $\varangle$-isometry. In order to prove this it is enough to see that $\psi$ preserves the spherical angle $\pi$ in both directions. But this is straightforward form the following equivalence, where we use the notation $\beta=\arccos \left(\frac{1}{3}\right)$ :

$$
\left(x^{(\beta)} \cup x^{(\pi)}\right) \cap\left(y^{(\beta)} \cup y^{(\pi)}\right)=\emptyset \text { and } y \in x^{(\beta)} \cup x^{(\pi)} \Longleftrightarrow y=-x .
$$

Case 4: when $\operatorname{dim} H=3$ and $0<\alpha<\frac{\pi}{3}$. Here we have $\operatorname{dim}(H \ominus[v])=2$ and therefore, unlike in the previous cases, we cannot apply Theorem 2. Instead, we shall utilise Lemma 4.5. As in Case 1, one gets that the transformation $\psi: S_{H \ominus[v]} \rightarrow$ $S_{H \ominus[v]}$ preserves the spherical angle $\beta:=\arccos \left(\frac{\cos \alpha}{1+\cos \alpha}\right) \in\left(\frac{\pi}{3}, \arccos \frac{1}{3}\right) \subset\left(\frac{\pi}{3}, \frac{\pi}{2}\right)$ in both directions. Thus $\psi$ also preserves the spherical angle $2 \beta \in\left(\frac{2 \pi}{3}, \pi\right)$ in both directions, which is a consequence of the following observation:

$$
\varangle(x, y)=2 \beta \Longleftrightarrow \#\left(x^{(\beta)} \cap y^{(\beta)}\right)=1 \quad\left(x, y, \in S_{H \ominus[v]}\right) .
$$

Therefore from (21) we conclude that $\phi$ preserves the quantum angle

$$
\gamma:=\arccos \left(\left|\cos ^{2} \alpha+\cos (2 \beta) \sin ^{2} \alpha\right|\right) \in\left(0, \frac{\pi}{2}\right]
$$

in both directions.

We proceed with examining the above quantum angle $\gamma$. An elementary calculation gives us the following:

$$
\begin{aligned}
\cos ^{2} \alpha+ & \cos (2 \beta) \sin ^{2} \alpha=\cos ^{2} \alpha+\left(2 \cos ^{2} \beta-1\right) \sin ^{2} \alpha \\
& =\cos ^{2} \alpha+\left(2\left(\frac{\cos \alpha}{1+\cos \alpha}\right)^{2}-1\right)\left(1-\cos ^{2} \alpha\right)=4 \cos \alpha+\frac{4}{\cos \alpha+1}-5 .
\end{aligned}
$$

Therefore (31) becomes

$$
\gamma=\arccos \left(\left|4 \cos \alpha+\frac{4}{\cos \alpha+1}-5\right|\right) .
$$


Next, an elementary calculation verifies the following inequality:

$$
2 c^{2}-1<4 c+\frac{4}{c+1}-5<c \quad\left(\frac{1}{2} \leq c<1\right) .
$$

It is also easy to show that for every $c \in\left[\frac{1}{2}, 1\right)$ we have

$$
4 c+\frac{4}{c+1}-5<0 \Longleftrightarrow \frac{1}{2} \leq c<\frac{1}{8}(1+\sqrt{17}),
$$

and

$$
4 c+\frac{4}{c+1}-5=0 \Longleftrightarrow c=\frac{1}{8}(1+\sqrt{17}) .
$$

Again, by elementary computations, we obtain

$$
\left|4 c+\frac{4}{c+1}-5\right|=5-4 c-\frac{4}{c+1}<1-2 c^{2} \leq c \quad\left(\frac{1}{2} \leq c<\frac{1}{8}(1+\sqrt{17})\right)
$$

which gives us

$$
2 c^{2}-1<\left|4 c+\frac{4}{c+1}-5\right|<c \quad\left(\frac{1}{2} \leq c<1\right) .
$$

Clearly, (32) and (33) implies $\gamma \in(\alpha, 2 \alpha) \cap\left(0, \frac{\pi}{2}\right]$.

Now, we consider four different possibilities. First, if $0<\alpha<\frac{\pi}{4}$, then $0<\gamma<\frac{\pi}{2}$, and by Lemmas 4.3 and 4.4 our map $\phi$ preserves the quantum angles $2 \alpha-\gamma \in\left(0, \frac{\pi}{2}\right)$ and $\gamma-\alpha \in\left(0, \frac{\pi}{2}\right)$ in both directions. Since one of them is less than or equal to $\frac{\alpha}{2}$, by a recursion we obtain a sequence of positive quantum angles converging to zero such that all of them is preserved by $\phi$ in both directions. Therefore by Lemma 4.5 we conclude that $\phi$ is a Wigner symmetry.

Second, by (32) we have $\gamma=\frac{\pi}{2}$ if and only if $\alpha=\arccos \frac{1+\sqrt{17}}{8}$. If this happens, then Uhlhorn's theorem implies that $\phi$ is a Wigner symmetry.

Third, we assume that $\frac{\pi}{4} \leq \alpha<\arccos \frac{1+\sqrt{17}}{8}$ is satisfied, and we claim that there exists a number $0<q<1$ such that we have $\gamma<(1+q) \alpha$ for every such $\alpha$. In fact, if this was not the case, then $\left|4 c+\frac{4}{c+1}-5\right|$ could be arbitrarily close to $2 c^{2}-1$ on the interval $\left[\frac{1+\sqrt{17}}{8}, \frac{1}{\sqrt{2}}\right]$. But then, continuity of the functions and compactness of the interval would imply that there is a number $c \in\left[\frac{1+\sqrt{17}}{8}, \frac{1}{\sqrt{2}}\right]$ at which these two functions attain the same value, contradicting to (33). Since by Lemma 4.3 the map $\phi$ preserves $\gamma-\alpha<q \alpha$, by a recursion we get a sequence of positive quantum angles $\left\{\alpha_{n}\right\}_{n=1}^{\infty}$ converging to zero such that they are preserved by $\phi$ in both directions. Therefore $\phi$ has to be a Wigner symmetry.

Finally, let us suppose that we have $\arccos \frac{1+\sqrt{17}}{8}<\alpha<\frac{\pi}{3}$. Since $\frac{\pi}{4} \leq$ $\arccos \frac{1+\sqrt{17}}{8}$, we get $\frac{\gamma}{\alpha}<\frac{\pi / 2}{\arccos \frac{1+\sqrt{17}}{8}}<1.8$ for every such $\alpha$. But $\phi$ preserves the quantum angle $\gamma-\alpha<0.8 \cdot \alpha$, therefore we have the following two possibilities. Either by a recursion we obtain a sequence of positive quantum angles converging to zero such that all of them is preserved by $\phi$ in both directions, or after some steps we conclude that $\phi$ preserves the quantum angle $\arccos \frac{1+\sqrt{17}}{8}$, and thus orthogonality in both directions. Both of them imply that $\phi$ is a Wigner symmetry.

Case 5: when $\operatorname{dim} H=3$ and $\frac{\pi}{3}<\alpha<\frac{\pi}{2}$. We claim that

$$
\measuredangle([x],[y])=\pi-2 \alpha \Longleftrightarrow \#\left([x]^{\alpha} \cap[y]^{\alpha}\right)=3 .
$$


In order to see this let $[x]$ and $[y]$ be two different lines and set $\gamma:=\measuredangle([x],[y]) \in$ $\left(0, \frac{\pi}{2}\right]$. There exist two orthogonal unit vectors $e_{1}, e_{2} \in H$ such that we have

$$
[x]=\left[\cos \frac{\gamma}{2} \cdot e_{1}+\sin \frac{\gamma}{2} \cdot e_{2}\right], \quad[y]=\left[\cos \frac{\gamma}{2} \cdot e_{1}-\sin \frac{\gamma}{2} \cdot e_{2}\right] .
$$

We fix a unit vector $e_{3}$ which is orthogonal to both $e_{1}$ and $e_{2}$. For $t_{1}, t_{2}, t_{3} \in \mathbb{R}$, $t_{1}^{2}+t_{2}^{2}+t_{3}^{2}=1$ we have

$$
\left[t_{1} \cdot e_{1}+t_{2} \cdot e_{2}+t_{3} \cdot e_{3}\right] \in[x]^{\alpha} \cap[y]^{\alpha}
$$

if and only if

$$
\cos \alpha=\left|t_{1} \cos \frac{\gamma}{2}+t_{2} \sin \frac{\gamma}{2}\right|=\left|t_{1} \cos \frac{\gamma}{2}-t_{2} \sin \frac{\gamma}{2}\right| .
$$

Therefore, we immediately conclude that either $t_{1}=0$, or $t_{2}=0$. In the first possibility we compute $\left|t_{2}\right|=\frac{\cos \alpha}{\sin \frac{\gamma}{2}}>0$, moreover, we have

$$
\frac{\cos \alpha}{\sin \frac{\gamma}{2}}<1 \Longleftrightarrow \gamma>\pi-2 \alpha
$$

and

$$
\frac{\cos \alpha}{\sin \frac{\gamma}{2}}=1 \Longleftrightarrow \gamma=\pi-2 \alpha .
$$

In the second possibility we have $\left|t_{1}\right|=\frac{\cos \alpha}{\cos \frac{\gamma}{2}} \in(0,1)$. Therefore if $\gamma<\pi-2 \alpha$, then $\#\left([x]^{\alpha} \cap[y]^{\alpha}\right) \leq 2$. Otherwise, we have

$$
\begin{aligned}
{[x]^{\alpha} \cap[y]^{\alpha}=\{} & {\left[\frac{\cos \alpha}{\sin \frac{\gamma}{2}} \cdot e_{2}+\sqrt{1-\frac{\cos ^{2} \alpha}{\sin ^{2} \frac{\gamma}{2}}} \cdot e_{3}\right],\left[\frac{\cos \alpha}{\sin \frac{\gamma}{2}} \cdot e_{2}-\sqrt{1-\frac{\cos ^{2} \alpha}{\sin ^{2} \frac{\gamma}{2}}} \cdot e_{3}\right], } \\
& {\left.\left[\frac{\cos \alpha}{\cos \frac{\gamma}{2}} \cdot e_{1}+\sqrt{1-\frac{\cos ^{2} \alpha}{\cos ^{2} \frac{\gamma}{2}}} \cdot e_{3}\right],\left[\frac{\cos \alpha}{\cos \frac{\gamma}{2}} \cdot e_{1}-\sqrt{1-\frac{\cos ^{2} \alpha}{\cos ^{2} \frac{\gamma}{2}}} \cdot e_{3}\right]\right\} . }
\end{aligned}
$$

Clearly, the third and fourth elements above are always different. Furthermore, they are also different from the first and second elements. Therefore we have $\#\left([x]^{\alpha} \cap[y]^{\alpha}\right)=3$ if and only if the first and second elements coincide, or equivalently, if $\cos \alpha=\sin \frac{\gamma}{2}$ which yields (34).

Now, by Case 4 and the following equivalence, $\phi$ is a Wigner symmetry:

$$
\begin{aligned}
\measuredangle([x],[y])=\pi-2 \alpha & \Longleftrightarrow \#\left([x]^{\alpha} \cap[y]^{\alpha}\right)=3 \Longleftrightarrow \#\left(\phi([x])^{\alpha} \cap \phi([y])^{\alpha}\right)=3 \\
& \Longleftrightarrow \measuredangle(\phi([x]), \phi([y]))=\pi-2 \alpha .
\end{aligned}
$$

Case 6: when $\operatorname{dim} H=3$ and $\alpha=\frac{\pi}{3}$. We claim that we have $\measuredangle([x],[y])=\frac{\pi}{2}$ if and only if $[x]^{\pi / 3} \cap[y]^{\pi / 3}=\left\{\left[u_{1}\right],\left[u_{2}\right],\left[u_{3}\right],\left[u_{4}\right]\right\}$ is a four-element set such that $\measuredangle\left(\left[u_{1}\right],\left[u_{3}\right]\right)=\measuredangle\left(\left[u_{1}\right],\left[u_{4}\right]\right)=\measuredangle\left(\left[u_{2}\right],\left[u_{3}\right]\right)=\measuredangle\left(\left[u_{2}\right],\left[u_{4}\right]\right)=\frac{\pi}{3}$ holds. Similarly as in the previous case, we have \# $\left([x]^{\pi / 3} \cap[y]^{\pi / 3}\right) \leq 3$ if and only if $\gamma \leq \frac{\pi}{3}$, otherwise \# $\left([x]^{\pi / 3} \cap[y]^{\pi / 3}\right)=4$ and $[x]^{\pi / 3} \cap[y]^{\pi / 3}=\left\{\left[v_{1}(\gamma)\right],\left[v_{2}(\gamma)\right],\left[v_{3}(\gamma)\right],\left[v_{4}(\gamma)\right]\right\}$ where we used the following notations:

$$
\begin{aligned}
& v_{1}(\gamma)=\frac{1 / 2}{\sin \frac{\gamma}{2}} \cdot e_{2}+\sqrt{1-\frac{1 / 4}{\sin ^{2} \frac{\gamma}{2}}} \cdot e_{3}, \quad v_{2}(\gamma)=\frac{1 / 2}{\sin \frac{\gamma}{2}} \cdot e_{2}-\sqrt{1-\frac{1 / 4}{\sin ^{2} \frac{\gamma}{2}}} \cdot e_{3}, \\
& v_{3}(\gamma)=\frac{1 / 2}{\cos \frac{\gamma}{2}} \cdot e_{1}+\sqrt{1-\frac{1 / 4}{\cos ^{2} \frac{\gamma}{2}}} \cdot e_{3}, \quad v_{4}(\gamma)=\frac{1 / 2}{\cos \frac{\gamma}{2}} \cdot e_{1}-\sqrt{1-\frac{1 / 4}{\cos ^{2} \frac{\gamma}{2}}} \cdot e_{3} .
\end{aligned}
$$


A straightforward computation gives the following:

$$
\begin{aligned}
\left\langle v_{1}(\gamma), v_{3}(\gamma)\right\rangle & =-\left\langle v_{1}(\gamma), v_{4}(\gamma)\right\rangle=-\left\langle v_{2}(\gamma), v_{3}(\gamma)\right\rangle \\
& =\left\langle v_{2}(\gamma), v_{4}(\gamma)\right\rangle=\sqrt{1-\frac{1 / 4}{\sin ^{2} \frac{\gamma}{2}}} \cdot \sqrt{1-\frac{1 / 4}{\cos ^{2} \frac{\gamma}{2}}} .
\end{aligned}
$$

Here $\sin ^{2} \frac{\gamma}{2}$ runs through the interval $\left(\frac{1}{4}, \frac{1}{2}\right]$, if $\gamma$ runs through $\left(\frac{\pi}{3}, \frac{\pi}{2}\right]$. Denoting $\sin ^{2} \frac{\gamma}{2}$ by $u$ and observing that the equation $\left(1-\frac{1}{4 u}\right)\left(1-\frac{1}{4(1-u)}\right)=\frac{1}{4}$ has the only solution $u=\frac{1}{2}$ verifies our claim.

Finally, similarly as in the previous case, one can show that $\phi$ preserves orthogonality in both directions, therefore $\phi$ has to be a Wigner symmetry.

Our final aim is to verify our Uhlhorn-type generalisation for complex spaces of dimension at least three. But before doing so, we need the following crucial lemma where we will use the notation

$$
\operatorname{diam}_{\measuredangle}\left([v]^{\alpha} \cap[w]^{\alpha}\right):=\sup \left(\measuredangle\left(\left[u_{1}\right],\left[u_{2}\right]\right):\left[u_{1}\right],\left[u_{2}\right] \in[v]^{\alpha} \cap[w]^{\alpha}\right) .
$$

Lemma 4.6. Let $0<\alpha<\frac{\pi}{4}$, $H$ be a complex inner product space with $\operatorname{dim} H \geq 3$, and $[v],[w] \in P(H)$ be two lines with $\measuredangle([v],[w])=: \gamma \in(0,2 \alpha)$. Then

$$
\operatorname{diam}_{\measuredangle}\left([v]^{\alpha} \cap[w]^{\alpha}\right)=2 \cdot \arccos \sqrt{\frac{\cos ^{2} \alpha-\sin ^{2}\left(\frac{\gamma}{2}\right)}{\cos ^{2}\left(\frac{\gamma}{2}\right)-\sin ^{2}\left(\frac{\gamma}{2}\right)}} .
$$

Moreover, there exist exactly two lines $\left[u_{1}\right],\left[u_{2}\right] \in[v]^{\alpha} \cap[w]^{\alpha}$ with $\measuredangle\left(\left[u_{1}\right],\left[u_{2}\right]\right)=$ $\operatorname{diam}_{\measuredangle}\left([v]^{\alpha} \cap[w]^{\alpha}\right)$; and if $0<\beta<\operatorname{diam}_{\measuredangle}\left([v]^{\alpha} \cap[w]^{\alpha}\right)$, then there exist at least three different lines $\left[u_{0}\right],\left[u_{1}\right],\left[u_{2}\right] \in[v]^{\alpha} \cap[w]^{\alpha}$ with $\measuredangle\left(\left[u_{0}\right],\left[u_{1}\right]\right)=\measuredangle\left(\left[u_{0}\right],\left[u_{2}\right]\right)=\beta$.

Proof. There is an orthonormal system $\left\{e_{1}, e_{2}\right\}$ such that we have

$$
[v]=\left[\cos \left(\frac{\gamma}{2}\right) \cdot e_{1}+\sin \left(\frac{\gamma}{2}\right) \cdot e_{2}\right] \text { and }[w]=\left[\cos \left(\frac{\gamma}{2}\right) \cdot e_{1}-\sin \left(\frac{\gamma}{2}\right) \cdot e_{2}\right] .
$$

Let us calculate the set $[v]^{\alpha} \cap[w]^{\alpha}$. For any $[u] \in P(H)$, we can find a vector $e_{3} \in H,\left\|e_{3}\right\|=1, e_{3} \perp e_{j}(j=1,2)$ and numbers $\lambda \in \mathcal{C}, \delta, \varepsilon \in\left[0, \frac{\pi}{2}\right]$ such that

$$
[u]=\left[\cos \delta \cdot e_{1}+\lambda \sin \delta \cos \varepsilon \cdot e_{2}+\sin \delta \sin \varepsilon \cdot e_{3}\right] .
$$

This line lies in $[v]^{\alpha} \cap[w]^{\alpha}$ if and only if we have $\cos \alpha=\left|\cos \left(\frac{\gamma}{2}\right) \cos \delta+\lambda \sin \left(\frac{\gamma}{2}\right) \sin \delta \cos \varepsilon\right|=\left|\cos \left(\frac{\gamma}{2}\right) \cos \delta-\lambda \sin \left(\frac{\gamma}{2}\right) \sin \delta \cos \varepsilon\right|$. The second equation implies that we have either $\delta=0$, or $\delta=\frac{\pi}{2}$, or $\varepsilon=\frac{\pi}{2}$ or $\lambda \in\{-i, i\}$. For the first one, we get $\gamma=2 \alpha$ which is impossible under our assumptions. Concerning the second one, the contradiction

$$
\frac{1}{\sqrt{2}}<\cos \alpha=\left|\sin \left(\frac{\gamma}{2}\right) \cos \varepsilon\right| \leq \sin \left(\frac{\gamma}{2}\right)<\sin \alpha<\frac{1}{\sqrt{2}}
$$

is yielded. The third case implies $\cos \delta=\frac{\cos \alpha}{\cos \left(\frac{\gamma}{2}\right)} \in(0,1)$. From the last possibility we obtain the following:

$$
\begin{aligned}
\cos ^{2} \alpha & =\cos ^{2}\left(\frac{\gamma}{2}\right) \cos ^{2} \delta+\sin ^{2}\left(\frac{\gamma}{2}\right) \sin ^{2} \delta \cos ^{2} \varepsilon \\
& =\cos ^{2}\left(\frac{\gamma}{2}\right) \cos ^{2} \delta+\sin ^{2}\left(\frac{\gamma}{2}\right)\left(1-\cos ^{2} \delta\right) \cos ^{2} \varepsilon,
\end{aligned}
$$

from which we conclude

$$
\cos \delta=\sqrt{\frac{\cos ^{2} \alpha-\sin ^{2}\left(\frac{\gamma}{2}\right) \cos ^{2} \varepsilon}{\cos ^{2}\left(\frac{\gamma}{2}\right)-\sin ^{2}\left(\frac{\gamma}{2}\right) \cos ^{2} \varepsilon}} .
$$


We note that the above fraction is positive, since we have $\cos \left(\frac{\gamma}{2}\right)>\cos \alpha>\frac{1}{\sqrt{2}}>$ $\sin \left(\frac{\gamma}{2}\right)$. We also point out that the $\varepsilon=\frac{\pi}{2}$ case in the present possibility covers the before mentioned third possibility. Therefore we conclude that

$$
\begin{aligned}
& {[v]^{\alpha} \cap[w]^{\alpha}=\{} {\left[\sqrt{\frac{\cos ^{2} \alpha-\sin ^{2}\left(\frac{\gamma}{2}\right) \cos ^{2} \varepsilon}{\cos ^{2}\left(\frac{\gamma}{2}\right)-\sin ^{2}\left(\frac{\gamma}{2}\right) \cos ^{2} \varepsilon}} \cdot e_{1}+\right.} \\
&+\lambda \sqrt{\frac{\cos ^{2}\left(\frac{\gamma}{2}\right)-\cos ^{2} \alpha}{\cos ^{2}\left(\frac{\gamma}{2}\right)-\sin ^{2}\left(\frac{\gamma}{2}\right) \cos ^{2} \varepsilon}} \cos \varepsilon \cdot e_{2} \\
&\left.+\sqrt{\frac{\cos ^{2}\left(\frac{\gamma}{2}\right)-\cos ^{2} \alpha}{\cos ^{2}\left(\frac{\gamma}{2}\right)-\sin ^{2}\left(\frac{\gamma}{2}\right) \cos ^{2} \varepsilon}} \sin \varepsilon \cdot e_{3}\right]:\left\|e_{3}\right\|=1, \\
&\left.e_{3} \perp e_{1}, e_{3} \perp e_{2}, 0 \leq \varepsilon \leq \frac{\pi}{2}, \lambda \in\{-i, i\}\right\} .
\end{aligned}
$$

Next, we verify (37). Since the function $g_{x, y}(t)=\frac{x-t}{y-t}$ is strictly decreasing on the interval $[0, x]$ if $0<x<y$, and strictly increasing on the interval $[0, y]$ if $0<y<x$, we estimate as follows:

$$
\begin{aligned}
\sup \left\{\measuredangle\left([u],\left[e_{1}\right]\right):\right. & \left.: u] \in[v]^{\alpha} \cap[w]^{\alpha}\right\} \\
& =\arccos \left(\inf \left\{\sqrt{\frac{\cos ^{2} \alpha-\sin ^{2}\left(\frac{\gamma}{2}\right) \cos ^{2} \varepsilon}{\cos ^{2}\left(\frac{\gamma}{2}\right)-\sin ^{2}\left(\frac{\gamma}{2}\right) \cos ^{2} \varepsilon}}: \varepsilon \in\left[0, \frac{\pi}{2}\right]\right\}\right) \\
& =\arccos \left(\min \left\{\sqrt{\frac{\cos ^{2} \alpha-t}{\cos ^{2}\left(\frac{\gamma}{2}\right)-t}}: t \in\left[0, \sin ^{2}\left(\frac{\gamma}{2}\right)\right]\right\}\right) \\
& =\arccos \sqrt{\frac{\cos ^{2} \alpha-\sin ^{2}\left(\frac{\gamma}{2}\right)}{\cos ^{2}\left(\frac{\gamma}{2}\right)-\sin ^{2}\left(\frac{\gamma}{2}\right)}}=\arccos \sqrt{\frac{1}{2} \cdot \frac{\cos ^{2} \alpha-\sin ^{2}\left(\frac{\gamma}{2}\right)}{\frac{1}{2}-\sin ^{2}\left(\frac{\gamma}{2}\right)}} \\
& \leq \arccos \sqrt{\frac{1}{2} \cdot \frac{\cos ^{2} \alpha-0}{\frac{1}{2}-0}}=\alpha<\frac{\pi}{4} .
\end{aligned}
$$

Notice that the left-hand side of (40) is strictly decreasing in $\gamma$. Moreover, the supremum is attained exactly when $\varepsilon=0$, in particular, if and only if we have either

$$
[u]=\left[\sqrt{\frac{\cos ^{2} \alpha-\sin ^{2}\left(\frac{\gamma}{2}\right)}{\cos ^{2}\left(\frac{\gamma}{2}\right)-\sin ^{2}\left(\frac{\gamma}{2}\right)}} \cdot e_{1}+i \cdot \sqrt{\frac{\cos ^{2}\left(\frac{\gamma}{2}\right)-\cos ^{2} \alpha}{\cos ^{2}\left(\frac{\gamma}{2}\right)-\sin ^{2}\left(\frac{\gamma}{2}\right)}} \cdot e_{2}\right] \in P_{\left[e_{1}\right],\left[e_{2}\right]},
$$

or

$$
[u]=\left[\sqrt{\frac{\cos ^{2} \alpha-\sin ^{2}\left(\frac{\gamma}{2}\right)}{\cos ^{2}\left(\frac{\gamma}{2}\right)-\sin ^{2}\left(\frac{\gamma}{2}\right)}} \cdot e_{1}-i \cdot \sqrt{\frac{\cos ^{2}\left(\frac{\gamma}{2}\right)-\cos ^{2} \alpha}{\cos ^{2}\left(\frac{\gamma}{2}\right)-\sin ^{2}\left(\frac{\gamma}{2}\right)}} \cdot e_{2}\right] \in P_{\left[e_{1}\right],\left[e_{2}\right]} .
$$

Now, we consider the following bijective linear isometry:

$$
R: H \rightarrow H, R\left(\lambda \cdot e_{1}+h\right)=\lambda \cdot e_{1}-h \quad\left(\lambda \in \mathbb{C}, h \in H, h \perp e_{1}\right),
$$

which clearly satisfies the equations $R([v])=[w], R([w])=[v]$ and $R\left([v]^{\alpha} \cap[w]^{\alpha}\right)=$ $[v]^{\alpha} \cap[w]^{\alpha}$. If $\left[u_{1}\right],\left[u_{2}\right] \in[v]^{\alpha} \cap[w]^{\alpha}$, then we have $\measuredangle\left(\left[u_{1}\right],\left[u_{2}\right]\right) \leq \measuredangle\left(\left[u_{1}\right],\left[e_{1}\right]\right)+$ 
$\measuredangle\left(\left[e_{1}\right],\left[u_{2}\right]\right)$. On the other hand, if $\left[\lambda \cdot e_{1}+h\right] \in[v]^{\alpha} \cap[w]^{\alpha}\left(h \perp e_{1},|\lambda|^{2}+\|h\|^{2}=1\right)$, then by (40) we have $|\lambda|>\frac{1}{\sqrt{2}}$, and thus we calculate

$$
\begin{array}{r}
\measuredangle\left(\left[\lambda \cdot e_{1}+h\right],\left[R\left(\lambda \cdot e_{1}+h\right)\right]\right)=\measuredangle\left(\left[\lambda \cdot e_{1}+h\right],\left[\lambda \cdot e_{1}-h\right]\right)=\left.\arccos || \lambda\right|^{2}-\|h\|^{2} \mid \\
=\left.\arccos |2 \cdot| \lambda\right|^{2}-1|=2 \cdot \arccos | \lambda \mid=2 \cdot \measuredangle\left(\left[\lambda \cdot e_{1}+h\right],\left[e_{1}\right]\right) .
\end{array}
$$

Therefore

$$
\begin{aligned}
\operatorname{diam}_{\measuredangle}\left([v]^{\alpha} \cap[w]^{\alpha}\right) & =2 \cdot \sup \left(\measuredangle\left([u],\left[e_{1}\right]\right):[u] \in[v]^{\alpha} \cap[w]^{\alpha}\right) \\
& =2 \cdot \arccos \sqrt{\frac{\cos ^{2} \alpha-\sin ^{2}\left(\frac{\gamma}{2}\right)}{\cos ^{2}\left(\frac{\gamma}{2}\right)-\sin ^{2}\left(\frac{\gamma}{2}\right)}},
\end{aligned}
$$

which verifies (37). Moreover, the above observations also imply that $\measuredangle\left(\left[u_{1}\right],\left[u_{2}\right]\right)=$ $\operatorname{diam}_{\measuredangle}\left([v]^{\alpha} \cap[w]^{\alpha}\right)$ and $\left[u_{1}\right],\left[u_{2}\right] \in[v]^{\alpha} \cap[w]^{\alpha}$ hold if and only if the lines $\left[u_{1}\right]$ and $\left[u_{2}\right]$ are exactly those defined in (41) and (42).

Finally, let $0<\beta<\operatorname{diam}_{\measuredangle}\left([v]^{\alpha} \cap[w]^{\alpha}\right)$. Let $[u]$ be the line defined in (41), and $[\widetilde{u}]$ be another line from $[v]^{\alpha} \cap[w]^{\alpha}$ such that $\measuredangle([u],[\widetilde{u}])=\beta$. The existence of such a line $[\widetilde{u}]$ is straightforward from the pathwise connectedness of $[v]^{\alpha} \cap[w]^{\alpha}$, which can be directly seen from (39). Since \# $\left([v]^{\alpha} \cap[w]^{\alpha} \cap P_{\left[e_{1}\right],\left[e_{2}\right]}\right)=2$, we obtain that $[\widetilde{u}] \notin P_{\left[e_{1}\right],\left[e_{2}\right]}$. Let $\widetilde{u}=\hat{u}+\check{u}$ such that $\hat{u} \in\left[e_{1}, e_{2}\right]$ and $\check{u} \perp e_{j}$ $(j=1,2)$. Clearly, by (39) the line $[\hat{u}-\check{u}]$ lies in $[v]^{\alpha} \cap[w]^{\alpha} \backslash\{[\widetilde{u}]\}$, moreover, we have $\measuredangle([u],[\hat{u}-\check{u}])=\measuredangle([u],[\widetilde{u}])=\beta$, which completes our proof.

We point out that if $\alpha=\frac{\pi}{4}$, then we have

$$
\operatorname{diam}_{\measuredangle}\left([v]^{\alpha} \cap[w]^{\alpha}\right)=\operatorname{diam}_{\measuredangle}\left([v]^{\alpha} \cap[w]^{\alpha} \cap P_{[v],[w]}\right)=\frac{\pi}{2}
$$

for any two different lines $[v],[w] \in P(H)$, which can be verified by utilising Bloch's representation. Therefore (37) does not hold for general quantum angles $\alpha$.

We conclude the paper by proving our result on complex projective spaces when the dimension of $H$ is at least three.

Proof of Theorem 4. Case 1: when $0<\alpha<\frac{\pi}{4}$. First, let us examine the following equation:

$$
\alpha=2 \cdot \arccos \sqrt{\frac{\cos ^{2} \alpha-\sin ^{2}\left(\frac{\gamma}{2}\right)}{\cos ^{2}\left(\frac{\gamma}{2}\right)-\sin ^{2}\left(\frac{\gamma}{2}\right)}} .
$$

It is clear that as $\gamma \rightarrow 0+$, the right-hand side tends to $2 \alpha$, and as $\gamma \rightarrow 2 \alpha-$, the limit of the right-hand side is zero. It was noted in the proof of Lemma 4.6 that the right-hand side is strictly decreasing. Therefore the above equation must have a unique solution $\gamma_{0}$ from the interval $(0,2 \alpha)$. Now, let us observe the following:

$$
\begin{aligned}
\alpha<2 \cdot \arccos \sqrt{\frac{\cos ^{2} \alpha-\sin ^{2}\left(\frac{\alpha}{2}\right)}{\cos ^{2}\left(\frac{\alpha}{2}\right)-\sin ^{2}\left(\frac{\alpha}{2}\right)}}=2 \cdot \arccos \sqrt{\frac{\cos ^{2} \alpha-\sin ^{2}\left(\frac{\alpha}{2}\right)}{\cos \alpha}} \\
\Longleftrightarrow \cos ^{2} \alpha-\sin ^{2}\left(\frac{\alpha}{2}\right)<\cos \alpha \cos ^{2}\left(\frac{\alpha}{2}\right) \Longleftrightarrow 0<\frac{1}{2} \sin ^{2} \alpha .
\end{aligned}
$$

Since the latter inequality is valid, we actually have $\alpha<\gamma_{0}<2 \alpha$. 
Next, by Lemmas 4.1 and 4.6 we infer the following equivalence-chain for every $[v],[w] \in P(H):$

$$
\begin{aligned}
\measuredangle([v],[w]) & =\gamma_{0} \Longleftrightarrow \operatorname{diam}_{\measuredangle}\left([v]^{\alpha} \cap[w]^{\alpha}\right)=\alpha \\
& \Longleftrightarrow\left\{\begin{array}{r}
\#\left([u]^{\alpha} \cap[v]^{\alpha} \cap[w]^{\alpha}\right) \leq 1 \text { for every }[u] \in[v]^{\alpha} \cap[w]^{\alpha}, \text { and } \\
\#\left\{[u] \in[v]^{\alpha} \cap[w]^{\alpha}: \#\left([u]^{\alpha} \cap[v]^{\alpha} \cap[w]^{\alpha}\right)=1\right\}=2,
\end{array}\right. \\
& \Longleftrightarrow\left\{\begin{array}{r}
\#\left([x]^{\alpha} \cap \phi([v])^{\alpha} \cap \phi([w])^{\alpha}\right) \leq 1 \text { for all }[x] \in \phi([v])^{\alpha} \cap \phi([w])^{\alpha}, \text { and } \\
\#\left\{[x] \in \phi([v])^{\alpha} \cap \phi([w])^{\alpha}: \#\left([x]^{\alpha} \cap \phi([v])^{\alpha} \cap \phi([w])^{\alpha}\right)=1\right\}=2,
\end{array}\right. \\
& \Longleftrightarrow \operatorname{diam}_{\measuredangle}\left(\phi([v])^{\alpha} \cap \phi([w])^{\alpha}\right)=\alpha \\
& \Longleftrightarrow \measuredangle(\phi([v]), \phi([w]))=\gamma_{0},
\end{aligned}
$$

i.e. $\phi$ preserves the quantum angle $\gamma_{0}$ in both directions. Lemma 4.4 implies that the quantum angle $2 \alpha$ is also preserved in both directions. Therefore, by Lemma 4.3 , our transformation preserves the quantum angles $\gamma_{0}-\alpha$ and $2 \alpha-\gamma_{0}$ in both directions. Since none of these quantum angles are zero and at least one of them is less than or equal to $\frac{\alpha}{2}$, a straightforward induction and Lemma 4.5 complete the proof of this case.

Case 2: when $\alpha=\frac{\pi}{4}$. First, if $[v] \perp[w]$, then by Lemma 4.1 we have

$$
[v]^{\pi / 4} \cap[w]^{\pi / 4}=\left\{\left[\sqrt{\frac{1}{2}} \cdot v+\lambda \sqrt{\frac{1}{2}} \cdot w\right]: \lambda \in \mathcal{C}\right\} .
$$

For arbitrary $\lambda, \mu \in \mathcal{C}$ we compute the following:

$$
\measuredangle\left(\left[\sqrt{\frac{1}{2}} \cdot v+\lambda \sqrt{\frac{1}{2}} \cdot w\right],\left[\sqrt{\frac{1}{2}} \cdot v+\mu \sqrt{\frac{1}{2}} \cdot w\right]\right)=\arccos \frac{|1+\lambda \bar{\mu}|}{2} .
$$

Therefore we conclude that \# $\left([u]^{\pi / 4} \cap[v]^{\pi / 4} \cap[w]^{\pi / 4}\right)=2$ is satisfied for every $[u] \in[v]^{\pi / 4} \cap[w]^{\pi / 4}$.

Next, we show that this property fails if $\measuredangle([v],[w]) \in\left(0, \frac{\pi}{2}\right)$. We will use the notations of (38). A direct calculation gives that the following set is a subset of $[v]^{\pi / 4} \cap[w]^{\pi / 4}:$

$$
\begin{aligned}
& M_{[v],[w]}:=\{[ \sqrt{\frac{\frac{1}{2}-\sin ^{2}\left(\frac{\gamma}{2}\right) \cos ^{2} \varepsilon}{\cos ^{2}\left(\frac{\gamma}{2}\right)-\sin ^{2}\left(\frac{\gamma}{2}\right) \cos ^{2} \varepsilon}} \cdot e_{1}+ \\
&+\lambda \sqrt{\frac{\cos ^{2}\left(\frac{\gamma}{2}\right)-\frac{1}{2}}{\cos ^{2}\left(\frac{\gamma}{2}\right)-\sin ^{2}\left(\frac{\gamma}{2}\right) \cos ^{2} \varepsilon}} \cos \varepsilon \cdot e_{2} \\
&\left.+\sqrt{\frac{\cos ^{2}\left(\frac{\gamma}{2}\right)-\frac{1}{2}}{\cos ^{2}\left(\frac{\gamma}{2}\right)-\sin ^{2}\left(\frac{\gamma}{2}\right) \cos ^{2} \varepsilon}} \sin \varepsilon \cdot e_{3}\right]:\left\|e_{3}\right\|=1, e_{3} \perp e_{1}, \\
&\left.e_{3} \perp e_{2}, 0 \leq \varepsilon \leq \frac{\pi}{2}, \lambda \in\{-i, i\}\right\} .
\end{aligned}
$$

In particular, we have

$$
[u]:=\left[\sqrt{\frac{\frac{1}{2}-\sin ^{2}\left(\frac{\gamma}{2}\right)}{\cos ^{2}\left(\frac{\gamma}{2}\right)-\sin ^{2}\left(\frac{\gamma}{2}\right)}} \cdot e_{1}+i \sqrt{\frac{\cos ^{2}\left(\frac{\gamma}{2}\right)-\frac{1}{2}}{\cos ^{2}\left(\frac{\gamma}{2}\right)-\sin ^{2}\left(\frac{\gamma}{2}\right)}} \cdot e_{2}\right] \in M_{[v],[w]}
$$


and

$$
\left[u^{\prime}\right]:=\left[\sqrt{\frac{\frac{1}{2}-\sin ^{2}\left(\frac{\gamma}{2}\right)}{\cos ^{2}\left(\frac{\gamma}{2}\right)-\sin ^{2}\left(\frac{\gamma}{2}\right)}} \cdot e_{1}-i \sqrt{\frac{\cos ^{2}\left(\frac{\gamma}{2}\right)-\frac{1}{2}}{\cos ^{2}\left(\frac{\gamma}{2}\right)-\sin ^{2}\left(\frac{\gamma}{2}\right)}} \cdot e_{2}\right] \in M_{[v],[w]} .
$$

A simple computation shows that $[u] \perp\left[u^{\prime}\right]$. Since $M_{[v],[w]}$ is pathwise connected, there exists a line $[\widetilde{u}] \in M_{[v],[w]}$ with $\measuredangle([\widetilde{u}],[u])=\frac{\pi}{4}$. As in the last paragraph of the proof of Lemma 4.6, we can see that $[\widetilde{u}] \notin P_{\left[e_{1}\right],\left[e_{2}\right]}$, and thus we can easily construct infinitely many other lines which are in $[v]^{\pi / 4} \cap[w]^{\pi / 4} \cap[u]^{\pi / 4}$. Therefore if two different lines $[v]$ and $[w]$ are not orthogonal, then there exists a line $[u] \in$ $[v]^{\pi / 4} \cap[w]^{\pi / 4}$ such that \# $\left([u]^{\pi / 4} \cap[v]^{\pi / 4} \cap[w]^{\pi / 4}\right)=\infty$.

By the above observations we have the following equivalence-chain:

$$
\begin{aligned}
{[v] } & \perp[w] \quad \Longleftrightarrow \#\left([u]^{\pi / 4} \cap[v]^{\pi / 4} \cap[w]^{\pi / 4}\right)=2 \text { for every }[u] \in[v]^{\pi / 4} \cap[w]^{\pi / 4} \\
& \Longleftrightarrow \#\left([x]^{\pi / 4} \cap \phi([v])^{\pi / 4} \cap \phi([w])^{\pi / 4}\right)=2 \text { for all }[x] \in \phi([v])^{\pi / 4} \cap \phi([w])^{\pi / 4} \\
& \Longleftrightarrow \phi([v]) \perp \phi([w]) .
\end{aligned}
$$

Therefore $\phi$ has to be a Wigner symmetry which completes the proof.

\section{ACKNOWLEDGEMENT}

The author was also supported by the "Lendület" Program (LP2012-46/2012)

of the Hungarian Academy of Sciences and by the Hungarian National Research, Development and Innovation Office - NKFIH (grant no. K115383).

\section{REFERENCES}

[1] J. Araujo, J.J. Font, Linear isometries between subspaces of continuous functions, Trans. Amer. Math. Soc. 349 (1997), 413-428.

[2] J. Araujo, J.J. Font, Linear isometries on subalgebras of uniformly continuous functions, Proc. Edinburgh Math. Soc. (2) 43 (2000), 139-147.

[3] J. Araujo, The noncompact Banach-Stone theorem, J. Operator Theory 55 (2006), 285-294.

[4] R. Baer, Linear Algebra and Projective Geometry, Academic Press, New York, 1952.

[5] V. Bargmann, Note on Wigner's theorem on symmetry operations, J. Math. Phys. 5 (1964) 862-868.

[6] F.S. Beckman and D.A. Quarles, On isometries of Euclidean spaces, Proc. Amer. Math. Soc. 4, (1953). 810-815.

[7] D.P. Blecher, L.E. Labuschagne, Logmodularity and isometries of operator algebras, Trans. Amer. Math. Soc. 355 (2003), 1621-1646 (electronic).

[8] F. Botelho, Isometries and Hermitian operators on Zygmund spaces, Canad. Math. Bull. 58 (2015), 241-249.

[9] F. Botelho, J. Jamison, and L. Molnár, Surjective isometries on Grassmann spaces, J. Funct. Anal. 265 (2013), 2226-2238.

[10] L. Bracci, G. Morchio, F. Strocchi, Wigner's theorem on symmetries in indefinite metric spaces, Commun. Math. Phys. 41 (1975), 289-299.

[11] P.M. Van den Broek, Symmetry transformations in indefinite metric spaces: A generalization of Wigner's theorem, Physica A 127 (1984), 599-612.

[12] M. Cambern, K. Jarosz, Multipliers and isometries in $H_{E}^{\infty}$, Bull. London Math. Soc. 22 (1990), 463-466.

[13] G. Chevalier, Wigner-type theorems for projections, Internat. J. Theoret. Phys. 47 (2008), 69-80.

[14] G. Chevalier, Wigner's Theorem and its Generalizations, Handbook of Quantum Logic and Quantum Structures, 429-475, Elsevier Sci. B. V., Amsterdam, 2007.

[15] U. Everling, Isometries of spheres, Proc. Amer. Math. Soc. 123 (1995), 2855-2859. 
[16] C.-A. Faure, An elementary proof of the fundamental theorem of projective geometry, Geom. Dedicata 90 (2002), 145-151.

[17] R.J. Fleming, J.E. Jamison, Isometries on Banach Spaces: Function Spaces, in: Chapman \& Hall/CRC Monographs and Surveys in Pure and Applied Mathematics, 129, Chapman \& Hall/CRC, Boca Raton, FL, 2003.

[18] R.J. Fleming, J.E. Jamison, Isometries on Banach spaces. Vol. 2. Vector-valued function spaces, in: Chapman \& Hall/CRC Monographs and Surveys in Pure and Applied Mathematics, 138, Chapman \& Hall/CRC, Boca Raton, FL, 2008.

[19] Gy.P. Gehér, An elementary proof for the non-bijective version of Wigner's theorem, Phys. Lett. A 378 (2014), 2054-2057.

[20] Gy.P. Gehér, P. Šemrl, Isometries of Grassmann spaces, J. Funct. Anal. 270 (2016), 15851601.

[21] M. Györy, Transformations on the set of all $n$-dimensional subspaces of a Hilbert space preserving orthogonality, Publ. Math. Debrecen 65 (2004), 233-242.

[22] J. Hou, L. Huang, Maps completely preserving idempotents and maps completely preserving square-zero operators, Israel J. Math. 176 (2010), 363-380.

[23] K. Jarosz, Any Banach space has an equivalent norm with trivial isometries, Israel J. Math. 64 (1988), 49-56.

[24] C.-K. Li, L. Plevnik, P. Šemrl, Preservers of matrix pairs with a fixed inner product value, Oper. Matrices 6 (2012), 433-464.

[25] J.S. Lomont, P. Mendelson, The Wigner unitary-antiunitary theorem, Ann. Math. 78 (1963) $548-559$.

[26] M. Martín, The group of isometries of a Banach space and duality, J. Funct. Anal. 255 (2008), 2966-2976.

[27] L. Molnár, Generalization of Wigner's unitary-antiunitary theorem for indefinite inner product spaces, Commun. Math. Phys. 201 (2000), 785-791.

[28] L. Molnár, Selected Preserver Problems on Algebraic Structures of Linear Operators and on Function Spaces, Springer-Verlag Berlin Heidelberg, 2007.

[29] L. Molnár, Transformations on the set of all $n$-dimensional subspaces of a Hilbert space preserving principal angles, Comm. Math. Phys. 217 (2001), 409-421.

[30] L. Molnár, Orthogonality preserving transformations on indefinite inner product spaces: generalization of Uhlhorn's version of Wigner's theorem, J. Funct. Anal. 194 (2002), 248-262.

[31] M.A. Nielsen and I.L. Chuang, Quantum Computation and Quantum Information, Cambridge University Press, Cambridge, 2000., 676 pp.

[32] J. Rätz, On Wigner's theorem: Remarks, complements, comments, and corollaries, Aequationes Math. 52 (1996) 1-9.

[33] L. Rodman, P. Šemrl, Orthogonality preserving bijective maps on finite dimensional projective spaces over division rings, Linear Multilinear Algebra 56 (2008), 647-664.

[34] L. Rodman, P. Šemrl, Preservers of generalized orthogonality on finite dimensional real vector and projective spaces, Linear Multilinear Algebra 57 (2009), 839-858.

[35] P. Šemrl, Applying projective geometry to transformations on rank one idempotents, J. Funct. Anal. 210 (2004), 248-257.

[36] P. Šemrl, Generalized symmetry transformations on quaternionic indefinite inner product spaces: an extension of quaternionic version of Wigner's theorem, Comm. Math. Phys. 242 (2003), 579-584.

[37] P. Šemrl, Maps on idempotent matrices over division rings, J. Algebra 298 (2006), 142-187.

[38] P. Šemrl, Orthogonality preserving transformations on the set of $n$-dimensional subspaces of a Hilbert space, Illinois J. Math. 48 (2004), 567-573.

[39] C.S. Sharma, D.L. Almeida, A direct proof of Wigner's theorem on maps which preserve transition probabilities between pure states of quantum systems, Ann. Phys. 197 (1990) 300-309.

[40] D. Sherman, Noncommutative $L^{p}$ structure encodes exactly Jordan structure, J. Funct. Anal. 221 (2005), 150-166.

[41] U. Uhlhorn, Representation of symmetry transformations in quantum mechanics, Ark. Fysik 23 (1963), 307-340.

[42] E.P. Wigner, Gruppentheorie und ihre Anwendung auf die Quantenmechanik der Atomspektrum, Fredrik Vieweg und Sohn, 1931. 
MTA-SZTe Analysis and Stochastics Research Group, Bolyai Institute, University of Szeged, H-6720 Szeged, Aradi vértanúk tere 1., Hungary

MTA-DE "Lendület" Functional Analysis Research Group, Institute of Mathematics, University of Debrecen, H-4010 Debrecen, P.O. Box 12, Hungary

Email address: gehergy@math.u-szeged.hu or gehergyuri@gmail.com

URL: http://www . math.u-szeged.hu/ gehergy/ 